\title{
JD11
}

\section{First Results from the FUSE Mission}

\author{
Chairperson: G. Sonneborn \\ Editors: G. Sonneborn \& H.W. Moos
}




\title{
First Results from the FUSE Mission
}

\author{
George Sonneborn \\ FUSE Project Scientist, NASA's Goddard Space Flight Center \\ Greenbelt, MD, USA. george.sonneborn@gsfc.nasa.gov \\ H. Warren Moos \\ FUSE Principal Investigator, Dept. of Physics and Astronomy \\ Johns Hopkins University, Baltimore, MD, USA. hwm@pha.jhu.edu
}

\begin{abstract}
The Far Ultraviolet Spectroscopic Explorer (FUSE) provides high-resolution spectroscopy at far ultraviolet (FUV) wavelengths (905$1187 \AA$ ) of many astronomical objects in order to address fundamental questions related to the origin of the universe. FUSE, a cooperative project of the United States, Canada, and France, was launched in June 1999 for a three-year primary mission. The instrument sensitivity permits studies of many extragalactic sources at high spectral resolution. The key science drivers for the mission design are absorption-line spectroscopy of the ISM and IGM to investigate $\mathrm{D} / \mathrm{H}, \mathrm{O}$ VI, and molecular hydrogen abundances and distribution in the Galaxy and beyond to $z \sim 0.3$. Most of the observing time is available to Guest Investigators. This Joint Discussion summarizes some of the scientific results determined primarily from the first several months of FUSE observations.
\end{abstract}

\section{Overview and Mission Status}

Presented by G. Sonneborn, NASA's Goddard Space Flight Center

The Far Ultraviolet Spectroscopic Explorer (FUSE) is a NASA astronomy mission that is exploring the universe from 905 to $1187 \AA$ with high spectral resolution and sufficient sensitivity for extragalactic absorption line studies. FUSE is a cooperative project of the U.S., Canadian, and French space agencies. The FUSE satellite was launched on 24 June 1999 on Delta II rocket for a three-year prime mission. The observing time is split equally between the FUSE Principal Investigator (PI) team and the Guest Investigator (GI) program during the prime mission. An extended mission, where all the observing time is for the GI program, has been approved by NASA for an additional two years.

This section provides a brief mission overview. A more detailed description is provided by Moos et al. (2000). The in-orbit performance of the instrument is given by Sahnow et al. (2000). Many of the scientific results described in this Joint Discussion first appeared in the 20 July 2000 issue of the Astrophysical Journal Letters. Other results were presented here for the first time. 
It has long been recognized that there are many important and unique spectral diagnostics in the FUSE bandpass. These include the Lyman series of $\mathrm{HI}$ and DI, the ground state electronic transitions of molecular hydrogen $\left(\mathrm{H}_{2}\right.$ and $\left.\mathrm{HD}\right)$, and resonance transitions of $\mathrm{O}$ VI and $\mathrm{S} \mathrm{VI}$, transitions that are formed by collisional ionization at $T \sim 300,000 \mathrm{~K}$. There are also resonance lines of many abundant elements at lower degrees of ionization, including $\mathrm{C}$ I-III, N I-III, O I, P II-V, S III-IV, Fe II-III, and Ar I. These lines provide a high density of strong transitions for interstellar medium (ISM) and intergalactic medium (IGM) studies, many more such lines than are available at longer UV or optical wavelengths.

Most ultraviolet instruments have not been able to obtain data on these important spectral features because it has been difficult to construct reflecting surfaces with high efficiency below $1200 \AA$. There have been a few exceptions, however. The rocket experiment of Carruthers (1970), which achieved the first detection of interstellar $\mathrm{H}_{2}$, the Copernicus satellite (e.g. Spitzer \& Jenkins 1975), the Hopkins Ultraviolet Telescope (e.g., Kruk et al. 1995); the Interstellar Medium Absorption Profile Spectrograph (IMAPS) experiment (e.g. Jenkins et al. 1989, 1999), and the ORFEUS (Orbiting and Retrievable Far and Extreme Ultraviolet Spectrometers) payload (e.g., Hurwitz et al. 1998a, de Boer et al. 1998; Richter et al. 1999). None of these instruments combined the far ultraviolet sensitivity, spectral resolving power, and mission duration of FUSE.

The primary science objectives of the FUSE mission are to study (1) the origin and evolution of the light elements (D abundances in the Galaxy and low redshift environments, the dependence of $\mathrm{D} / \mathrm{H}$ on Galactic chemical evolution, and He II absorption at $2.0<z<3.0$ ), (2) the physical processes controlling the evolution of galaxies (circulation of matter throughout the Galaxy, the effect of Local Group gas on the evolution of the Milky Way, and the study of hot and cold phases of the ISM via $\mathrm{O}$ VI and $\mathrm{H}_{2}$ ), and (3) the origin and evolution of stars and planetary systems (stellar mass loss and interaction with the ISM, coronae of solar-type stars, and the formation and evolution of proto-planetary disks).

In the FUSE mission concept, the PI is responsible for mission design, development, operations. FUSE uses an efficient and innovative optical design with four independent optical channels. This provides high throughput and redundancy. The optical coatings are optimized for wavelength coverage. The spectra are recorded by two delay-line microchannel plate detectors. Mission and science operations are under the control of the PI team at Johns Hopkins University (Baltimore, USA). FUSE data has a short proprietary period (6 months) that encourages rapid publication of results.

Following an in-orbit checkout and science verification phase, flight and ground systems meet mission performance requirements. Regular science operations started in December 1999. Some scheduling constraints were adopted to improve observing efficiency and to maintain channel coalignment. On-target science efficiency is now averaging $\sim 30 \%$ over the past few months. The instrument sensitivity meets pre-launch predictions. The spectral resolution is $\lambda / \Delta \lambda \sim 20,000$ over most of the bandpass. The detector background levels are low $\left(<10^{-5}\right.$ counts pixel $\left.{ }^{-1} \mathrm{sec}^{-1}\right)$. Far ultraviolet scattered light is extremely low. 
For further information the reader is invited to consult the FUSE mission web site at http://fuse.pha.jhu.edu. FUSE science data is available through its public archive at http://archive.stsci.edu/fuse. Information about the FUSE GI program is available at http://fusewww.gsfc.nasa.gov/fuse/.

\section{Deuterium}

Presented by A.Vidal-Madjar, Institut d'Astrophysique de Paris. alfred@iap.fr

Deuterium is believed to be produced in significant amounts only during primordial Big Bang nucleosynthesis (BBN) and thoroughly destroyed in stellar interiors. It is thus a key element in cosmology and in Galactic chemical evolution (see e.g. Audouze \& Tinsley 1976; Boesgaard \& Steigman 1985; Olive et al. 1990; Pagel et al. 1992; Vangioni-Flam \& Cassé 1994, 1995; Prantzos 1996; Scully et al. 1997; Cassé \& Vangioni-Flam 1998, 2000).

The first measurement of the interstellar atomic $\mathrm{D} / \mathrm{H}$ ratio (Rogerson \& York 1973) was $(\mathrm{D} / \mathrm{H})_{I S M} \simeq 1.4 \pm 0.2 \times 10^{-5}$. Subsequent observations have shown that this value is representative of the present epoch in the vicinity of the Sun. For more than a decade, this interstellar deuterium abundance has been used to constrain BBN in a direct way. However, abundances measured at lower metallicities should reflect the actual primordial value. For this reason the deuterium abundance is now being studied in high redshift QSO absorbers.

\subsection{Lines of sight toward QSOs}

The point of this approach is that one may probe low metallicity systems, and potentially provide direct access to the primordial value, $\mathrm{D} / \mathrm{H}_{0}$. Studies of lines of sight toward three QSOs (Burles \& Tytler 1998a, 1998b; O'Meara et al., in preparation) lead to a possible range of $2.4-4.8 \times 10^{-5}$ for $\mathrm{D} / \mathrm{H}_{0}$. From these values, the corresponding baryon density evaluated in the context of standard BBN model of the universe $\left(\Omega_{\mathrm{b}} h^{2}\right.$, see Burles et al. 1999; Nollett \& Burles 2000) was found to be $\Omega_{\mathrm{b}} h^{2}=0.019 \pm 0.002$. A recent estimate from cosmic microwave background (CMB) observations (e.g. Jaffe et al. 2000) is $\Omega_{\mathrm{b}} h^{2}=0.032 \pm 0.005$, and the two results are close to disagreement.

Explanations may be found in the cosmological models (see e.g. Kaplinghat \& Turner 2000). However, note that $\mathrm{D} / \mathrm{H}$ measurements made toward a low redshift QSO (D/H being possibly larger than $10^{-4}$; Webb et al. 1997; Tytler et al. 1999) corresponds to an even stronger potential disagreement, since then $\Omega_{\mathrm{b}} h^{2}$ should be smaller than 0.01 . Thus it is important to investigate in more detail the possibility of varying $\mathrm{D} / \mathrm{H}$ ratios in different astrophysical environments (e.g. Lemoine et al. 1999). It is crucial to understand the cause of such spatial variations in $\mathrm{D} / \mathrm{H}$ in order to deduce a more reliable value of the primordial $\mathrm{D} / \mathrm{H}$ abundance ratio.

\subsection{Interstellar observations}

Several methods have been used to measure the interstellar abundance of deuterium. Among them, the most straightforward has used the Lyman series of D I and $\mathrm{HI}$ in the far-UV in absorption against the background continuum of hot 
stars. These observations have been performed using the Copernicus satellite, the International Ultraviolet Explorer, the Hubble Space Telescope, and now FUSE. Note that short duration Space Shuttle instruments like ORFEUS, and particulary IMAPS, have also contributed to these investigations.

Cool stars The main advantage of observing cool stars is that they can be selected nearby. This results in low H I column densities, and "simple" lines of sight. In this technique the DI and H I ISM features are observed in absorption against stellar emission lines. One of the difficulties inherent to this approach is that the detailed structure of the line of sight has been found only through the observation of the Fe II and $\mathrm{Mg}$ II ions. In particular, important species like $\mathrm{N}$ I and O I cannot be studied (see below). Moreover, the background chromospheric Lyman $\alpha$ emission line has to be modeled. Such a procedure potentially introduces systematic errors.

Nevertheless, the analysis of GHRS spectra of Capella has provided the most precise measurement of the local $\mathrm{D} / \mathrm{H}$ ratio (Linsky et al. 1995), due in part to the relative simplicity of the line of sight: $\mathrm{D} / \mathrm{H}_{\text {Capella }}=1.60 \pm 0.09_{-0.10}^{+0.05} \times 10^{-5}$.

White dwarfs Observing white dwarfs has many advantages for studying the local ISM. They can be chosen near the Sun, circumventing the main disadvantage of OB stars. In the HST regime, the N I triplet at $1200 \AA$ as well as the O I $1302 \AA$ are available, allowing an accurate assessment of the line of sight velocity structure.

Such observations toward G191-B2B were conducted using both HST high resolution spectrographs (Lemoine et al. 1996; Vidal-Madjar et al. 1998; Sahu et al. 1999; Vidal-Madjar 2000). The line of sight velocity structure, consistent in many spectral lines (about 15, including Ly $\alpha, \mathrm{N} \mathrm{I}, \mathrm{O}$ I, Si II, Mg II, Fe II, and Si III), indicates the presence of one $\mathrm{H}$ I region - the local interstellar cloud (LIC) that is also observed toward Capella - together with a more complex and ionized $\mathrm{H}$ II region that has a two-component velocity structure.

Although the results of Vidal-Madjar et al. (1998) for G191-B2B were contested by Sahu et al. (1999), Vidal-Madjar (2000) showed that there is general agreement between independent analyses for this sight line. If the $\mathrm{D} / \mathrm{H}$ ratio for the LIC, common to the G191-B2B and Capella sight-lines (these stars are separated by only $8^{\circ}$ on the sky), is forced to be that found toward Capella, then the $\mathrm{D} / \mathrm{H}$ ratio for the other components has to be significantly lower (less than $1.0 \times 10^{-5}$ ). Forcing a single $\mathrm{D} / \mathrm{H}$ ratio in all three components leads, on the contrary, to a much lower "average" $\mathrm{D} / \mathrm{H}$ ratio of $1.2 \pm 0.1 \times 10^{-5}$. Using $\mathrm{O} I$ as a tracer for $\mathrm{HI}_{\mathrm{I}}$ leads to the same conclusion. FUSE observations in the direction of G191-B2B are expected to further clarify the nature of the $\mathrm{D} / \mathrm{H}$ abundance on this sight line.

$O B$ stars $O B$ stars are located further away than the white dwarfs studied for $\mathrm{D} / \mathrm{H}$ in the local ISM and therefore sample larger amounts of gas over greater distances. This also introduces more complexity to the line of sight velocity structure. On the other hand, OB stars often have very smooth stellar continua because of large stellar rotation. It is the observation of higher Lyman lines in OB stars within $500 \mathrm{pc}$ that forms the basis of the $\mathrm{D} / \mathrm{H}$ results from Copernicus and IMAPS. The velocity structure of $\mathrm{H} \mathrm{I}$ on the line of sight is best defined by 
NI and O I (Ferlet 1981, York et al. 1983, Meyer et al. 1997, Meyer et al. 1998), ions that were shown to be good tracers of $\mathrm{HI}$ in the ISM and that have many transitions in the FUSE range.

From published values, it seems that $\mathrm{D} / \mathrm{H}$ ranges from $\mathrm{D} / \mathrm{H}_{I S M} \sim 5 \times 10^{-6}$ to $\mathrm{D} / \mathrm{H}_{I S M} \sim 4 \times 10^{-5}$. This large range could represent differences in the $\mathrm{D} / \mathrm{H}$ ratio within the local ISM as large as a factor $\sim 4$ over scales as small as a few hundred parsecs. This possibility is strongly supported by two new $\mathrm{D} / \mathrm{H}$ measurements from IMAPS. Jenkins et al. (1999) found a low value (D/H $\left.=7.4_{-1.5}^{+2.0} \times 10^{-6}\right)$ toward $\delta$ Orionis while toward $\gamma^{2}$ Velorum Sonneborn et al. (2000) found a high value $\left(\mathrm{D} / \mathrm{H}=2.18_{-0.31}^{+0.36} \times 10^{-5}\right)$. The quoted errors are $90 \%$ confidence limits.

Therefore, the essential question is: do these variations really exist since the number of well studied sightlines in the different domains remains relatively small?

In order to make some progress, many more $\mathrm{D} / \mathrm{H}$ measurements are necessary to confirm these results and to identify the responsible processes. This is an important purpose of the FUSE observatory mission.

\subsection{FUSE}

In recent months the FUSE satellite has obtained many observations to study the distribution of deuterium in the ISM. Their analysis is underway, so it is premature to report any results at the time of this Joint Discussion. From these studies we plan to evaluate the present day deuterium abundance in a wide variety of locations, possibly linked to the past star formation rate as well as to the hypothesized infall of less processed gas in our Galaxy, and thus better understand Galactic chemical evolution.

FUSE was designed to allow the evaluation of the deuterium abundance in many lines of sights, ranging from:

- the local ISM in the direction of white dwarfs and cool stars;

- the more distant ISM and nearby Galactic halo in the direction of hot sub-dwarfs;

- the Galactic disk over several kiloparsecs in the direction of $O$ and early B stars;

- the more distant halo, the high velocity cloud complexes as well as intergalactic clouds in the direction of low redshift QSOs, AGNs and blue compact galaxies.

During the FUSE mission lifetime we expect to gather many new $\mathrm{D} / \mathrm{H}$ measurements in many different astrophysical environments.

The first $\mathrm{D} / \mathrm{H}$ measurements from FUSE should be available soon since the deuterium lines are clearly seen toward at least five stars in the local ISM and two distant disk stars. Several of the Lyman lines in the FUSE spectrum of one of these, the $\mathrm{O}$ subdwarf $\mathrm{BD}+28^{\circ} 4211$, are shown in Figure 1. 


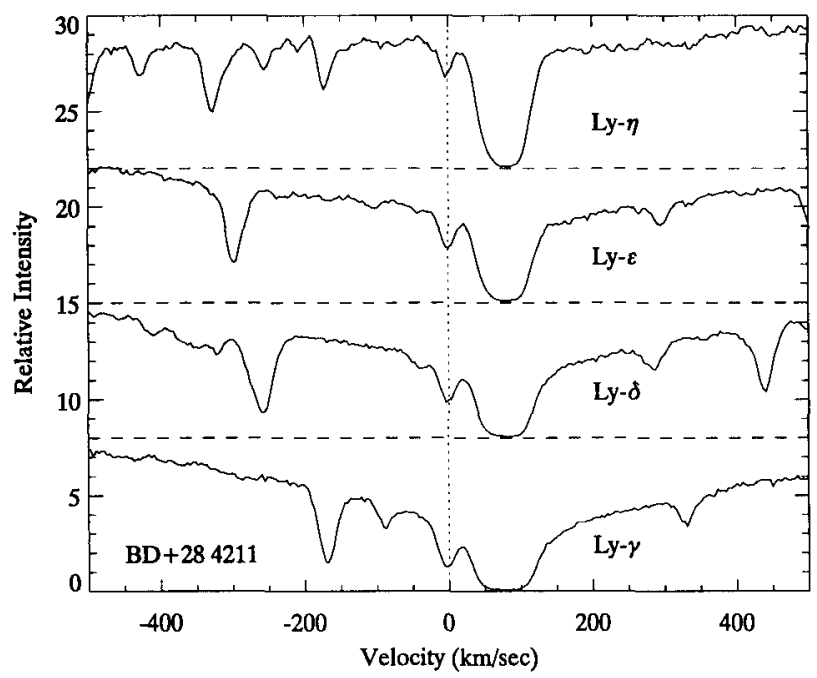

Figure 1. Portions of a FUSE spectrum of $B D+28^{\circ} 4211$ show the interstellar features near Lyman $\gamma, \delta, \epsilon$, and $\eta$. The horizontal dashed lines mark the zero level for each line. The zero point of the velocity scale is set centered on the interstellar D I features (vertical dashed line). The $\mathrm{S} / \mathrm{N}$ in this LWRS observation is about 100 per resolution element.

\subsection{Conclusions}

The present status may be summarized as follows. The different - and discordant $-\mathrm{D} / \mathrm{H}$ measurements in the Milky Way may imply a large range in $\mathrm{D} / \mathrm{H}$ within $500 \mathrm{pc}$ of the Sun. It is possible that not all measurements are correct; some as yet unknown systematic errors may be identified.

If the variations of the $\mathrm{D} / \mathrm{H}$ ratio in the local ISM are shown to be insignificant, then one could quote as an average of the published values: $(\mathrm{D} / \mathrm{H})_{I S M} \simeq$ $1.3 \pm 0.4 \times 10^{-5}$. The rather large uncertainty arises from a conservative treatment of the errors.

On the contrary, if $\mathrm{D} / \mathrm{H}$ does vary in the ISM, as indicated by recent studies, one has to understand the processes responsible. Until then, no measurement of the atomic $D / H$ ratio in the ISM or the IGM should be considered reliable. Moreover, one should expect the actual abundance variations in the interstellar gas to be larger in reality than the average values measured over long lines of sight. Our hope is that the FUSE mission will provide a major step toward resolving these problems. 


\section{Hot Galactic Halo}

Presented by B. D. Savage, Dept. of Astronomy, University of Wisconsin. savage@astro.wisc.edu

The existence of a hot interstellar medium (ISM) in the Milky Way and other galaxies has widespread ramifications for understanding the chemical evolution of stellar systems, the distribution of metals inside and outside of galaxies, and the cycles of matter and energy between stars and the material out of which they form. The hot ISM in the Milky Way was first detected in the 1970s through X-ray observations of thermally-excited emission from nearby $10^{6} \mathrm{~K}$ gas (Williamson et al. 1994) and by Copernicus observations of interstellar O VI absorption in clouds located within $\sim 1 \mathrm{kpc}$ of the Sun (York 1974; Jenkins 1978a, 1978b). These observations transformed our view of the ISM from one which was rather quiescent and cool to one which was dynamic, evolving, and possibly permeated by a hot substrate. This shift rekindled interest in decades-old suggestions that a corona of hot gas might envelop the Galaxy, and it sparked further theoretical work to explain the complex picture of the ISM that continues to evolve until this day.

Far-ultraviolet observations of O VI absorption and emission provide new information about the physical processes controlling the evolution of the hot plasmas. Of the ions accessible at wavelengths $\lambda>912 \AA, \mathrm{O} V \mathrm{I}$ is the best tracer of gas at coronal temperatures $\left(T \sim 10^{5}-10^{6} \mathrm{~K}\right)$. Unlike lower ionization species observable by HST (e.g., Si IV or C IV), O vi cannot be readily produced by photoionization by stars (114 eV photons are required). O vi peaks in abundance in collisional ionization equilibrium at temperatures $T \approx 3 \times 10^{5} \mathrm{~K}$ compared to $(0.8,1.0,2.0) \times 10^{5} \mathrm{~K}$ for Si IV, CIV, and N V, respectively (Sutherland \& Dopita 1993).

Furthermore, O VI traces gas near the peak of the cooling curve, where the gas evolves rapidly, and spans the temperature regime between X-ray emitting gas $\left(T>10^{6} \mathrm{~K}\right)$ and lower ionization material observable in the near-ultraviolet, optical, infrared, and radio regimes. When the line profiles can be observed at spectral resolutions sufficient to study individual components along a sight line, O VI observations provide information about the line-of-sight distribution of the hot gas that X-ray emission or low resolution spectroscopy cannot provide because of foreground/background confusion.

In the pioneering theoretical paper "On a Possible Interstellar Galactic Corona," Spitzer (1956) discussed the physical basis for a hot interstellar gas phase extending away from the Galactic plane into the halo. The hot gas was required to provide the pressure support for the intermediate velocity clouds seen in the spectra of halo stars in the absorption lines of $\mathrm{Ca}$ II.

Except for brief observing programs by HUT and the ORFEUS spectrographs (Hurwitz \& Bowyer 1996; Hurwitz et al. 1998b; Widmann et al. 1999; Sembach, Savage, \& Hurwitz 1999), we have been unable to obtain information about O VI in the Galactic halo until the launch of FUSE in 1999.

FUSE observations of $\mathrm{O}$ VI absorption through the entire Galactic halo toward 11 AGNs have been reported by Savage et al. (2000). The observations were obtained with the objects centered in the large $30^{\prime \prime} \times 30^{\prime \prime}$ aperture of the 
LiF1 channel. The spectroscopic reductions followed the basic data handling procedures discussed by Sembach et al. (2000). The spectra have a velocity resolution of approximately $25 \mathrm{~km} \mathrm{~s}^{-1}$ (FWHM).

The weaker $\mathrm{O}$ VI $\lambda 1037.62$ line is near strong absorption by $\mathrm{C} \mathrm{II}^{*} \lambda 1037.02$ and the $\mathrm{H}_{2}(5-0) \mathrm{R}(1)$ and $\mathrm{P}(1)$ lines at 1037.15 and $1038.16 \AA$. The stronger $\mathrm{O}$ vi $\lambda 1031.93$ line is usually relatively free of blending with other species since the nearby $\mathrm{H}_{2}(6-0) \mathrm{R}(3)$ and $\mathrm{R}(4)$ lines at 1031.19 and $1032.36 \AA$ are often weak and well-separated in velocity from O VI. The contamination of the O VI $\lambda 1037.62$ line is severe. Sample spectra on a velocity basis for the O VI $\lambda 1031.93$ line are illustrated in Figure 2 for seven AGNs.

The O VI $\lambda 1031.93$ absorption is so broad that it is nearly fully resolved by FUSE. Therefore, the observed absorption line profiles were converted into measures of O VI apparent column density per unit velocity, $\mathrm{N}_{a}(v)$, through the relation $\mathrm{N}_{a}(v)$ [ions $\mathrm{cm}^{-2}\left(\mathrm{~km} \mathrm{~s}^{-1}\right)^{-1}$ ] $=m_{e} c / \pi e^{2} \tau_{a}(v)(f \lambda)^{-1}=3.768 \times 10^{14}$ $\tau_{a}(v)(f \lambda)^{-1}$, where $f=0.133$ is the oscillator strength of the $\lambda 1031.93$ line, $\lambda$ is the wavelength in $\AA$, and $\tau_{a}(v)$ is the apparent absorption optical depth (see Savage \& Sembach 1991). The continuum levels are well-defined by the smooth flux distributions provided by the AGNs. For cases where the O VI $\lambda 1037.62$ line could be measured, Savage et al. found the same values of $\log \mathrm{N}_{a}(v)$ near maximum absorption as those obtained for the stronger $\lambda 1031.93$ line. This implies there is no evidence for unresolved saturated structure in the $\mathrm{OVI}$ absorption.

The O VI absorption profiles illustrated in Figure 2 are often complex and trace a wide range of phenomena in or near the Milky Way. Those portions of the profiles between approximately -100 and $+100 \mathrm{~km} \mathrm{~s}^{-1}$ are likely tracing gas in the thick O VI halo of the Milky Way. The higher velocity portions of the profiles provide information about $\mathrm{O}$ VI absorption in the high velocity clouds (see the next section).

Total column densities of O vi through the complete Galactic halo coupled with estimates of the mid-plane space density of $\mathrm{O}$ VI, $n_{0}$, can be used to obtain information about the distribution of O VI away from the plane of the Galaxy. Assuming an exponential gas distribution with $n(|z|)=n_{0} \exp (-|z| / h)$, where $h$ is the O VI scale height, it follows that the O VI column density perpendicular to the plane for an object with latitude $b$ is given by $\mathrm{N}(\mathrm{O} \mathrm{VI}) \sin |b|=n_{0} h[1-$ $\exp (-|z| / h)]$. Here $\mathrm{N}(\mathrm{O}$ vI) is the line-of-sight column density to an object at a distance $|z|$ away from the plane. For extragalactic objects, where $|z| \gg h$, $\mathrm{N}(\mathrm{O} \mathrm{VI}) \sin |b|=n_{0} h$.

Values of $\log [\mathrm{N}(\mathrm{O} \mathrm{VI}) \sin |b|]$ toward 11 extragalactic objects observed by FUSE range from 13.80 to 14.64 . The median $\log [\mathrm{N}(\mathrm{O} \vee \mathrm{VI}) \sin |b|]$ in the sample is 14.21 . The irregular nature of the absorption is revealed by the 0.84 dex range in $\log [\mathrm{N}(\mathrm{O}$ vI $) \sin |b|]$.

From the Copernicus observations of $\mathrm{O}$ VI absorption toward hot stars, Jenkins (1978b) estimated a mid-plane density $n_{0}=2.8 \times 10^{-8} \mathrm{~cm}^{-3}$. However, that estimate assumed a small scale height $(0.3 \mathrm{kpc})$ for the $\mathrm{O}$ VI absorbing layer. The new FUSE observations imply the scale height must be about 10 times larger. Therefore, a new estimate of $n_{0}$ from the Copernicus O VI survey was obtained that is appropriate for a large scale height absorbing layer. The result is $n_{0}=$ $2.0 \times 10^{-8} \mathrm{~cm}^{-3}$. 
For an O VI mid-plane density of $2.0 \times 10^{-8} \mathrm{~cm}^{-3}$, the FUSE measurements imply scale heights ranging from 1.0 to $7.0 \mathrm{kpc}$. The median and average values are 2.6 and $2.9 \mathrm{kpc}$, respectively. Edgar \& Savage (1989) devised an analysis procedure for estimating scale heights that allows for the irregular distribution of the gas. Adopting their procedure and using the 11 FUSE values of $\mathrm{N}(\mathrm{O} \mathrm{vI})$ $\sin |b|$, the FUSE data imply $h(\mathrm{O} \mathrm{vI})=2.7 \pm 0.4 \mathrm{kpc}$, where the listed $1 \sigma$ errors do not include systematic errors.

The O VI scale height of $2.7 \pm 0.4 \mathrm{kpc}$ can be compared with the values of $h=5.1 \pm 0.7,4.4 \pm 0.6$, and $3.9 \pm 1.4 \mathrm{kpc}$ for Si IV, C IV, and NV, respectively, determined by Savage et al. (1997). The more confined distributions of O VI and $\mathrm{NV}$ compared to $\mathrm{CIV}$ are also apparent in plots of $\mathrm{N}(\mathrm{CIV}) / \mathrm{N}(\mathrm{O} \mathrm{VI})$ and $\mathrm{N}(\mathrm{C}$ IV $) / \mathrm{N}(\mathrm{Nv})$ versus $|z|$ toward objects in the disk and halo. Spitzer (1996) noted that the value of $\mathrm{N}(\mathrm{CIV}) / \mathrm{N}(\mathrm{O} \mathrm{VI})$ increases from $\sim 0.15$ in the disk to 0.9 for objects in the low halo. Values of $N(\mathrm{CIV})$ have been measured using HST for five AGNs. Combining these with the values of $\mathrm{N}(\mathrm{O}$ VI $)$ measured by FUSE yields $\mathrm{N}(\mathrm{C}$ IV $) / \mathrm{N}(\mathrm{O}$ vi $)=0.58,0.63,0.45,0.63$ and 1.74 , respectively, for the five extragalactic objects. The increase in this ratio by about a factor of 4 from the disk to the typical extragalactic halo sight line implies a large change in the ionization state of the highly ionized gas as a function of distance from the Milky Way plane.

The FUSE observations of strong O VI absorption toward 10 of 11 extragalactic objects implies the widespread existence of hot gas in the halo of the Milky Way as predicted by Spitzer (1956). The decreasing scale heights in the sequence Si IV, CIV, Nv, to O VI provides information about the changing ionization state of the gas with distance from the Galactic plane.

Reviews of the theories for the origins of the highly ionized atoms in the ISM are found in Spitzer $(1990,1996)$ and Sembach, Savage, \& Tripp (1997). The primary types of theories involve conductive heating $(\mathrm{CH})$ where cool gas evaporates into an adjacent hot medium, radiative cooling (RC) where hot gas cools as it flows into the halo or down onto the disk, and turbulent mixing layers (TML) where hot and cool gas are mixed through turbulence (Slavin, Shull, $\&$ Begelman 1993). The different models make predictions for the expected values of the various ionic ratios. The $\mathrm{CH}$ models are compatible with the value of $\mathrm{N}(\mathrm{C} \mathrm{IV}) / \mathrm{N}(\mathrm{O} \mathrm{VI}) \sim 0.15$ found at low $|z|$, while the values of $\sim 0.6$ found toward the extragalactic objects observed by FUSE are better explained by a combination of the RC and TML models.

Shull \& Slavin (1994) developed a hybrid model for the highly ionized gas in the Galactic halo in order to explain the smaller scale height of $\mathrm{N} v$ compared to Si IV and CIV. In their model the highly ionized ions at low $|z|$ are produced mainly in isolated SNRs while those at high $|z|$ are mainly found in radiatively cooling superbubbles that break through the disk producing Rayleigh-Taylor instabilities and turbulent mixing layers. Confirmation that the scale height difference (smaller scale heights for ions with higher ionization potentials) first seen for N V and C IV is also clearly present in the new FUSE O VI measurements suggests that such hybrid models offer substantial promise for explaining the origin of the highly ionized species in the Galactic halo. Another example of a hybrid model is that of Ito \& Ikeuchi (1988) which includes the cooling gas of a Galactic fountain flow (Shapiro \& Field 1976) to provide the hot collisionally 
ionized gas and photoionization by the extragalactic background (Hartquist, Pettini, \& Tallant 1984; Fransson \& Chevalier 1985) to assist in the production of Si IV and Crv. The ionizing photons might also be provided by hot white dwarfs (Dupree \& Raymond 1983). The new observations with FUSE imply several processes may be required to achieve a more complete understanding of the origins of the low density highly ionized gas extending away from the Galactic plane.

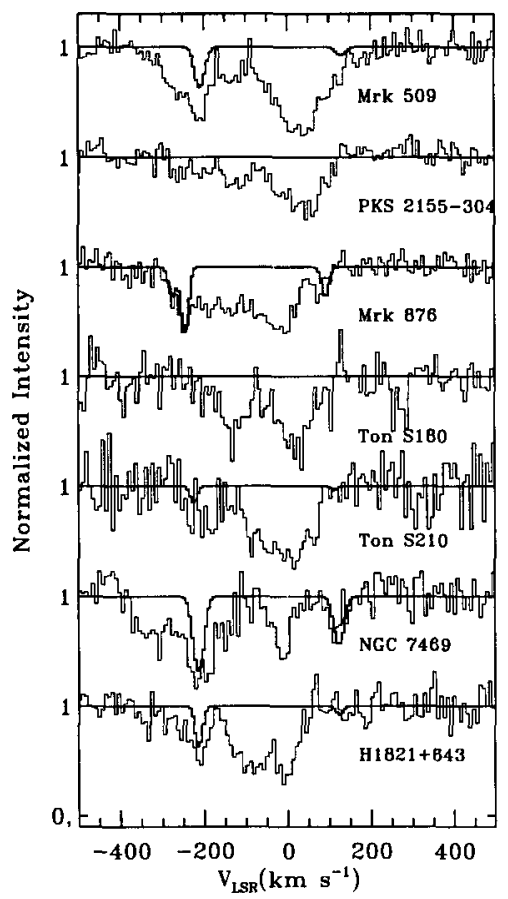

Figure 2. Normalized intensity versus LSR velocity for the $\mathrm{O}$ VI $\lambda 1031.93$ line along 7 HVC sight lines. The heavy solid line overplotted on each spectrum is a model of the $\mathrm{H}_{2}$ absorption in the (6-0) $\mathrm{P}(3) \lambda 1031.19$ and $\mathrm{R}(4)$ $\lambda 1032.35$ lines. O VI absorption arising in the low Galactic halo is confined mainly to the velocity range between -100 and +100 $\mathrm{km} \mathrm{s}^{-1}$ (except for $\mathrm{H} 1821+643$, where the halo range extends to about $-180 \mathrm{~km} \mathrm{~s}^{-1}$ ). The remainder of the absorption at high velocities $\left(v_{L S R} \approx-350\right.$ to -100 $\mathrm{km} \mathrm{s}^{-1}$ ) is due to O vr in HVCs. The detections of $\mathrm{O}$ VI indicate that the HVCs contain hot, collisionally ionized gas at temperatures of $10^{5}-10^{6} \mathrm{~K}$ (adapted from Sembach et al. 2000).

\section{High Velocity Clouds}

Presented by K. R. Sembach, Dept. of Physics and Astronomy, Johns Hopkins University.sembach@pha.jhu.edu

Most of the interstellar gas within the Milky Way has a bulk velocity consistent with rotation about the center of the Galaxy. However, many high velocity clouds (HVCs) have peculiar velocities that are at least $50-100 \mathrm{~km} \mathrm{~s}^{-1}$ in excess of those expected for corotating gas. Explaining their existence has remained an outstanding challenge for several decades since no single model of their kinematics can account for the motions of all of the known HVCs (see Wakker \& van Woerden 1997). A variety of processes are probably responsible for distributing and circulating this high velocity gas. Suggested mechanisms include supernova-driven "galactic fountains" (Shapiro \& Field 1976; Bregman 1980), ram pressure or tidal stripping of material from the Magellanic Clouds (Moore \& Davis 1994; Lin et al. 1995), infalling gas from outside the Milky Way (Oort 
1970; Mirabel \& Morras 1984), and identification of HVCs as "primordial" remnants from the formation of the Local Group (Blitz et al. 1999).

Distinguishing among these possibilities requires information about the elemental abundances, physical properties, and distances of the HVCs. The abundances and physical conditions of the clouds can be assessed directly through UV absorption-line spectroscopy by looking for the absorption they produce when viewed against the light of background halo stars and UV-bright extragalactic objects such as quasars and active galactic nuclei. FUSE is a powerful new observatory that is providing new insight into the origins of HVCs. The combination of its high sensitivity and high spectral resolution makes it an ideal instrument for observing HVCs within the Milky Way and Local Group.

\subsection{Hot Gas in HVCs}

Understanding the physical conditions and ionization of HVCs is a key step toward constructing a complete description of the high velocity gas. This is particularly important because recent studies with the Hubble Space Telescope (Sembach et al. $1995 ; 1999$ ) have shown that some HVCs are probably located in the Local Group since they have ionization properties indicative of exposure to the extragalactic radiation field. Furthermore, HVCs known to lie closer to the Galactic plane have been observed through their $\mathrm{H} \alpha$ emission, indicating that they too contain ionized gas (Tufte et al. 1998; Bland-Hawthorn et al. 1998).

The large peculiar velocities of HVCs make them excellent candidates for studying the hot gas within the clouds and their surrounding environments. A prime diagnostic of collisionally ionized gas at temperatures $T \sim 10^{5}-10^{6} \mathrm{~K}$ is the O VI $\lambda \lambda 1031.93,1037.62$ doublet, which can be observed in the spectra of distant QSOs and AGNs with FUSE. The presence of O VI in HVCs shows that these clouds are much more complex than previously thought.

HVCs close to the Galactic plane $(<3 \mathrm{kpc})$ are likely to originate in the fountain flow established by supernova explosions. However, others lie at considerable distances from the Galactic plane; these include the Magellanic Stream system and Local Group HVCs. FUSE has discovered O VI in several HVCs located at large distances from the Galactic plane (e.g., Complex C), as well as the Magellanic Stream (Sembach et al. 2000). These O vi detections are shown in Figure 2. They imply that hot $\left(T \sim 3 \times 10^{5} \mathrm{~K}\right)$, collisionally-ionized gas is an important constituent of the HVCs since O VI is difficult to produce by photoionization unless the gas density is extremely low and the path lengths over which the absorption occurs are very large $(l>100 \mathrm{kpc})$. The association of $\mathrm{O}$ VI with HI HVCs in several cases suggests that the O VI is produced at interfaces or mixing layers between the $\mathrm{H}_{\mathrm{I}}$ clouds and hot $\left(T \sim 10^{6} \mathrm{~K}\right)$, low density $\left(n_{H}<10^{-4}-10^{-5} \mathrm{~cm}^{-3}\right)$ gas in the Galactic corona or Local Group. Such a medium would be extremely difficult to detect by other means. Alternately the O VI may originate within cooling regions of hot gas clouds situated in the Local Group or in the halo of the Galaxy.

In at least one case, non-detection of high velocity O VI along a sight line near an HVC indicates that the ionized boundaries of some HVCs may be closely related to the neutral gas boundaries. Richter et al. (2000) studied the FUSE spectrum of PG 0804+761, which lies near to HVC Complex A. They find no 
O VI or neutral gas absorption at the velocity of Complex A $\left(v_{L S R} \sim-180\right.$ $\mathrm{km} \mathrm{s}^{-1}$ ) and deduce that Complex $\mathrm{A}$ has a well-defined, sharp edge in this direction.

\subsection{Abundances}

FUSE is providing new information about the elemental abundances and dust content of HVCs. Murphy et al. (2000) analyzed FUSE spectra of Mrk 876, a Seyfert 1 galaxy behind HVC Complex C. This HVC is located well above the Galactic plane (>10 kpc) and is probably a combination of low-metallicity material falling onto the Milky Way and enriched material spewed into the halo from the Galactic disk. The HVC is detected in several atomic species, including $\mathrm{CII}, \mathrm{NI}, \mathrm{NII}$, and FeII. The ratio $\mathrm{N}(\mathrm{Fe} I \mathrm{I}) / \mathrm{N}(\mathrm{HI})$ is particularly interesting because it is only $0.5 \pm 0.2$ times the solar $\mathrm{Fe} / \mathrm{H}$ ratio. This could imply that the metallicity of Complex $\mathrm{C}$ along this sight line is higher than the metallicity of 0.1 solar found by Wakker et al. (1999a) for the Mrk 290 sight line using HST measurements of S II. However, recent work by Gibson et al. (2000) indicates that several other Complex $\mathrm{C}$ sight lines observed by HST also imply a low ( $~ 0.1-0.3$ solar) metallicity, so it is more likely that the high Fe II/H I ratio observed for the Mrk 876 sight line is due to the presence of ionized gas in the HVC. This conclusion is supported by the FUSE detections of N II and O VI at similar velocities to that of the Fe II. The non-detection of Fe III by FUSE along the Mrk 876 sight line probably indicates that most of the Fe II observed occurs in neutral rather than ionized gas; the amount of Fe in dust grains must be a small percentage of the total $\mathrm{Fe}$ abundance, especially if the HVC is infalling material enriched by gas circulated up into the halo from the Galactic disk.

The detection of low metallicity gas in Complex $\mathrm{C}$ flowing onto the Galaxy has major implications for the chemical evolution of the Milky Way. A galaxywide low metallicity gas mass infall rate of approximately $1 M_{\odot} \mathrm{yr}^{-1}$ is required to solve the long standing "G-dwarf problem" relating to the unusual metallicity distribution found in long-lived $\mathrm{G}$ stars. Complex $\mathrm{C}$ alone provides an inflow per unit area at about the required rate. If five to ten objects like Complex $\mathrm{C}$ exist over the entire Galaxy, the G-dwarf problem is solved (Wakker et al. 1999a). The identification of Complex $\mathrm{C}$ as a low-metallicity, low-dust cloud suggests that the assembly of the Milky Way is a process that continues to the present day.

FUSE observations of high velocity cloud HVC $287.5+22.5+240$ in the direction of NGC 3783 have helped to constrain the possible origins for another distant HVC. The HVC has a metallicity of $0.2-0.4$ solar, similar to that of the Small Magellanic Cloud (Lu et al. 1998; Sembach et al. 2001). The relative abundance pattern for the elements studied by FUSE ( $\mathrm{Si}, \mathrm{P}, \mathrm{Fe}$ ) also resembles that of warm gas in the SMC. The kinematics, and position of HVC 287.5+22.5+240 indicate that the HVC is part of the Leading Arm of the Magellanic Stream. The tidal plus weak drag model proposed by Gardiner (1999) shows that the material in the Leading Arm was stripped primarily from the disk of the SMC and is located a distance of about 50-75 kpc from the Sun. This feature is observed in sensitive H I $21 \mathrm{~cm}$ maps of the Magellanic Stream (Putman et al. 1998). A detailed study of the high velocity $\mathrm{H}_{\mathrm{I}} 21 \mathrm{~cm}$ emission in the direction of NGC 3783 at different angular resolutions by Wakker et al. (1999b) reveals 
that discrete structures exist on scales down to $1^{\prime}(=14.5 \mathrm{pc}$ at a distance $\mathrm{D}=$ $50 \mathrm{kpc}$ ). They estimate that the HVC is part of an ensemble of small gas clouds with internal thermal pressures $P / k=1.1 n_{H} T \sim 360 \mathrm{~cm}^{-3} \mathrm{~K}$ for an assumed distance of $50 \mathrm{kpc}$.

\subsection{Molecular Gas}

Most previous studies of molecular gas in high velocity clouds have been limited to searches for $\mathrm{CO}$ emission that have resulted in many non-detections (see Wakker et al. 1997; Akeson \& Blitz 1999). While valuable for assessing whether the clouds contain large quantities of molecules, these searches are relatively insensitive to diffuse gas with $N\left(\mathrm{H}_{2}\right)<10^{18} \mathrm{~cm}^{-2}$. Recent FUSE observations of the high velocity cloud HVC $287.5+22.5+240$ demonstrate that it is possible for molecular hydrogen to exist in HVCs (Sembach et al. 2001). The amount of molecular hydrogen is small compared to atomic hydrogen $\left(N\left(\mathrm{H}_{2}\right) \approx 6 \times 10^{16}\right.$ $\left.\mathrm{cm}^{-2}, f_{\mathrm{H}_{2}}=2 N\left(\mathrm{H}_{2}\right) /\left[2 N\left(\mathrm{H}_{2}\right)+N(\mathrm{H} \mathrm{I})\right] \sim 1.6 \times 10^{-3}\right)$, but it is easily detected in more than 30 lines arising in rotational levels $J=0$ to $J=3$. An additional detection of $\mathrm{H}_{2}$ in the high velocity gas between the Galaxy and Large Magellanic Cloud has been made by Richter et al. (1999) using ORFEUS. That cloud also has a relatively low molecular hydrogen fraction, about half of the value observed for HVC $287.5+22.5+240$.

The existence of molecular gas in HVC $287.5+22.5+240$ requires that either the $\mathrm{H}_{2}$ formed in situ or that molecules formed within the SMC survived tidal stripping. This HVC also contains dust grains ( $\mathrm{Lu}$ et al. 1998). In Complex C, where there is no detectable $\mathrm{H}_{2}$ absorption, there is also no evidence for dust (Murphy et al. 2000). Thus, it appears that presence of dust and $\mathrm{H}_{2}$ are related in HVCs in the sense that when one is present, so is the other. Additional studies of other HVCs will be required to determine whether this relationship holds in general.

\section{Warm and Hot Ionized Gas in the Galactic Disk}

Presented by E. B. Jenkins, Princeton University Observatory. ebj@astro.princeton.edu

This discussion will start with the Local Interstellar Medium (LISM), a complex of warm, partly ionized clouds of gas (Frisch 1995; Gry 1996; Lallement 1996; Linsky 1996) immersed within a much hotter, fully ionized medium called the Local Bubble (LB). The LB, which we will discuss next, is an irregularly shaped cavity of very hot $\left(T \sim 10^{6} \mathrm{~K}\right)$ X-ray emitting gas that extends to about 100 pc away from the Sun (Cox \& Reynolds 1987; Snowden et al. 1990, 2000; Snowden 1998; Sfeir et al. 1999; Welsh et al. 1999). On a much grander scale, the disk of our Galaxy holds many other regions of hot gas, perhaps similar to the LB. FUSE can investigate the properties of partly and fully ionized gases over all three scales.

\subsection{Ionization of the LISM}

Our Solar System is situated within one of the partly ionized clouds within the LB (Lallement \& Bertin 1992; Redfield \& Linsky 2000). The physical properties 
of this gas (density, temperature and ionization), as deduced from clues that arise from its interaction with the Sun (Quémerais et al. 1994), seem approximately consistent with those of other clouds in the LB (Lallement \& Ferlet 1997; Wood \& Linsky 1997; Ferlet 1999; Jenkins, Gry, \& Dupin 2000). An important topic is the partial ionization of the material. Vallerga (1998) has calculated that the intensity of ionizing radiation from $\epsilon$ and $\beta$ CMa plus many nearby white dwarf stars is sufficient to explain the degree of ionization of $\mathrm{H}(\sim 25 \%$ and somewhat variable), but the even larger fractional ionization of $\mathrm{He}(\sim 35 \%$ and relatively constant) (Dupuis et al. 1995; Wolff, Koester, \& Lallement 1999) remains a mystery. Two principal hypotheses have been advanced to explain the helium ionization: (1) equilibrium photoionization arising from EUV radiation emitted by conduction fronts at the interfaces between the clouds and the surrounding, much hotter material (Slavin 1989) or (2) an incomplete recovery from a much more highly ionized condition created by a supernova shock in the recent past (Lyu \& Bruhweiler 1996).

We have employed FUSE observations of the absorption lines in the spectra of nearby, bright white dwarf stars to gain further insights on the source of ionization of the LISM (Jenkins et al. 2000). The three elements N, O and Ar all have transitions from the ground states of their neutral forms in the FUSE wavelength band. These elements are unlikely to have any significant depletion onto dust grains (Meyer, Jura, \& Cardelli 1996; Meyer, Cardelli, \& Sofia 1997; Sofia \& Jenkins 1998), but their susceptibilities to being photoionized are very different from that of hydrogen. Figure 3 shows that in a medium that is partially ionized by photons, neutral $\mathrm{He}, \mathrm{N}, \mathrm{O}$ and Ar have photoionization cross sections divided by their ions' respective rates of recombinations with free electrons that are significantly larger than those of $\mathrm{H}$.

As we consider the significance of the curves displayed in Fig. 3, it is important not to overlook the importance of charge exchange reactions with hydrogen. The rate constant $\sim 10^{-9} \mathrm{~cm}^{3} \mathrm{~s}^{-1}$ for oxygen atoms (Field \& Steigman 1971) is so much larger than the recombination rate with free electrons, $\left(5 \times 10^{-13} \mathrm{~cm}^{3} \mathrm{~s}^{-1}\right.$ at $T=7000 \mathrm{~K}$ ), that we can be certain that the ionization of $\mathrm{O}$ is virtually locked to that of $\mathrm{H}$. For $\mathrm{N}$ the situation is similar, although its charge exchange rate of $8 \times 10^{-13} \mathrm{~cm}^{3} \mathrm{~s}^{-1}$ (Butler \& Dalgarno 1979) is about equal to the recombination rate. By contrast, the charge exchange rate of $\mathrm{Ar}$ is only $\sim 10^{-14} \mathrm{~cm}^{3} \mathrm{~s}^{-1}$, and hence its equilibrium is determined simply by the ionization rate divided by the local electron density.

Table 1 shows the deficiencies relative to neutral hydrogen, expressed as a logarithmic comparison with B star abundances, of the neutral forms of the three elements in the directions of 4 white dwarf stars observed with FUSE. [We employ the term "deficiencies" in favor of "depletions" because the latter is commonly used to describe the effect of elements condensing into solid form on dust grains.] Oxygen shows a mild deficiency which is not far from what is seen elsewhere in the medium where the densities are high (and ionization is low), so we can surmise that there are no extraordinary departures of the oxygen ionization from that of hydrogen, as expected. By contrast, if we make the reasonable assumption that the deficiencies of $\mathrm{N}$ and $\mathrm{Ar}$ are caused by their 


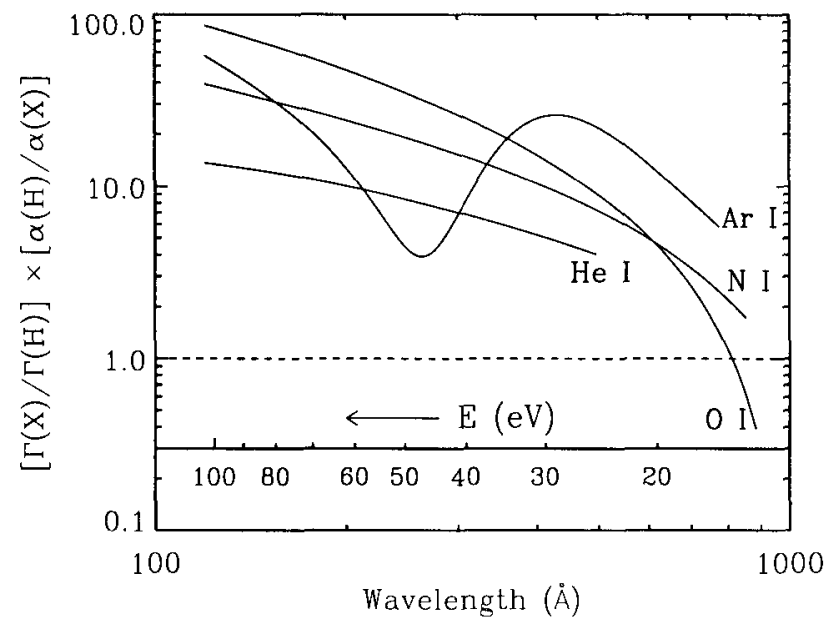

Figure 3. A comparison, as a function of photon energy, of the ionization cross sections of various elements in their neutral forms divided by the recombination rates of their singly-ionized forms at $T=7000 \mathrm{~K}$, compared to that of hydrogen.

being in unseen stages of ionization ${ }^{1}$, we can infer that indeed we are witnessing their greater susceptibility to photoionization by some EUV radiation field of uncertain origin. In the case where we were seeing left-over ionization from a once hot gas that is cooling radiatively, we would expect $N(\operatorname{ArI}) / N(\mathrm{HI})$ to be very close to the total $\mathrm{Ar} / \mathrm{H}$ (Shapiro \& Moore 1976).

The fact that the deficiency of $\mathrm{N}$ is greater than Ar toward two of the stars in the sample is puzzling. For a radiation field that is distributed over a broad range of energies, we would expect Ar to show a stronger effect, even near the edges of the cloud where the fractional ionization is high (Jenkins et al. 2000). We will briefly revisit this problem in $§ 5.2$.

\subsection{Hot $\mathrm{Gas}$ in the Local Bubble}

An important objective of FUSE is to observe absorption by the $\lambda \lambda 1032,1038$ doublet of OVI. While this ion was the subject of an extensive survey with the Copernicus satellite to determine the distribution and nature of gas that is collisionally ionized at $T \sim 3 \times 10^{5} \mathrm{~K}$ (Jenkins $1978 \mathrm{a}, \mathrm{b}$ ), most of the target stars that were bright enough to observe with Copernicus were all well beyond the perimeter of the LB. FUSE observations of white dwarf stars provide, for

\footnotetext{
${ }^{1}$ The singly ionized forms of $\mathrm{N}$ and Ar have transitions in the FUSE band. Unfortunately, the white dwarf stars were observed before FUSE was aligned in a way that all spectral channels could record the spectrum of a point source simultaneously. The $1084 \AA$ transition of N II was outside the wavelength range of the available channel. The transition of $\mathrm{Ar}$ II at $920 \AA$ is weak and hard to untangle from high members of the Lyman series of $\mathrm{H} \mathbf{~}$.
} 
Table 1. Deficiencies ${ }^{a}$ of Neutral Forms of Elements Relative to Hydrogen

\begin{tabular}{c|cccc}
\hline & G191- & & WD & WD \\
Species & B2B & GD 394 & $2211-495$ & $2331-475$ \\
\hline O I & -0.19 & -0.38 & -0.11 & -0.15 \\
N I & -0.26 & -0.60 & -0.54 & -0.12 \\
Ar I & $<-0.42$ & -0.45 & -0.43 & -0.37 \\
\hline
\end{tabular}

${ }^{a}$ Expressed for element $\mathrm{X}$ in the form $\log N(\mathrm{X} \mathrm{I}) / N(\mathrm{H} \mathrm{I})-\log (\mathrm{X} / \mathrm{H})_{\mathrm{B} \text { stars }}$.

the first time ever, an opportunity to sample the LB and possible O VI-bearing conductive interfaces not far from the Sun.

So far, we have not detected much O VI within the LB. Oegerle, et al. (in preparation) occasionally have registered weak, broad features consistent with $N(\mathrm{OVI}) \sim 10^{13} \mathrm{~cm}^{-2}$. However toward many different stars there is a puzzling absence of narrow O VI lines that are expected to arise, at the very least, from the conductive interface around the cloud within which the Sun is immersed. If there is no transverse magnetic field to inhibit the conduction, Slavin (1989) predicts that $N(\mathrm{O} \mathrm{VI})=10^{13} \mathrm{~cm}^{-2}$, which should create a feature with a $10 \%$ depth if $b=20 \mathrm{~km} \mathrm{~s}^{-1}$. Such a feature seems to be absent in most of the cases examined so far. While the inhibiting effect of a magnetic field seems like an attractive solution to this problem, the fact that the Voyager 1 spacecraft has not yet passed through solar wind termination shock puts an increasingly harder constraint on the local magnetic field strength (Lallement 1998), assuming that the field direction is not parallel to the Sun's motion. [A strong, tangled magnetic field would also solve the problem of the large disparity between the local thermal pressure $p / k=1700 \mathrm{~cm}^{-3} \mathrm{~K}$ and one that is about ten times larger outside (Berghöfer et al. 1998).]

One possible solution for the lack of O VI surrounding the local cloud is to adopt the model for the LB proposed by Breitschwerdt \& Schmutzler (1994). In their model, one or more explosive events within a dense cloud creates an expansion that is rapid enough to make adiabatic cooling the dominant process, resulting in final temperatures of order $T \sim 50,000 \mathrm{~K}$, but with an ionization state characteristic of much hotter gas. Recombination radiation is then the dominant source of the observed diffuse, soft X-ray background. This is an attractive way to overcome the problem that FUSE does not observe narrow $\mathrm{O}$ VI features toward white dwarf stars (and it also solves the pressure imbalance problem discussed above). Also, the dominant source of radiation is from the helium recombination radiation at $54.5 \mathrm{eV}$ and slightly above. This energy is within the narrow range where $\mathrm{N}$ can be more easily photoionized than $\mathrm{Ar}$ (see Fig. 3), and thus the helium recombination radiation might explain why the $\mathrm{N}$ deficiency sometimes exceeds that of Ar (see Table 1). Nevertheless, a troubling counterargument is that the expected column density of $\mathrm{O} V \mathrm{VI}$ to the edge of the LB should be of order $10^{14} \mathrm{~cm}^{-2}$ (Breitschwerdt, private communication), a value 


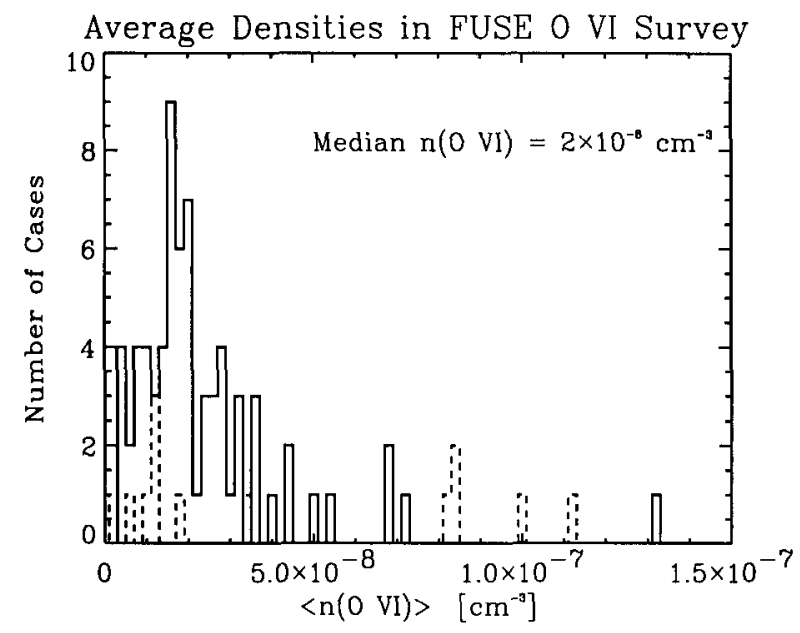

Figure 4. Frequencies of occurrences of average densities of O VI, $N(\mathrm{O} \mathrm{VI}) /$ distance, for stars observed by FUSE so far. No corrections for the presence an overlapping HD feature have yet been made. Dashed bars indicate upper limits.

far greater than the detections and upper limits for broader features observed up to now.

\section{3. $O$ VI in the Plane of the Galaxy}

An ambitious survey of O VI is being carried out by FUSE for slightly more than one hundred stars in the plane and halo of our Galaxy. Reports on the results for targets away from the plane and extragalactic sources are reported elsewhere in this article (§3. and §4.). Presently, the survey for the plane is about half complete. Figure 4 shows the distribution of average densities over the different lines of sight. The median value of $n(\mathrm{O} \mathrm{VI})=2 \times 10^{-8} \mathrm{~cm}^{-3}$ is very close to the value obtained from the Copernicus survey (Jenkins 1978b). The dispersion of values is significant however. Most of the outlyers at the high density end of the distribution arise from targets that are behind or within regions that are otherwise known to be excited by stellar winds or other energetic phenomena (examples: stars near $\eta$ Carinae, a star behind the Vela SNR, and another behind the Mon-Gem Loop).

A study of the dispersion of O VI densities will form the basis for an improved understanding on the statistical properties of the $\mathrm{O}$ VI-bearing material. Already, it is apparent that this dispersion does not seem to decrease with increasing distance, as one might expect from randomly distributed regions that had just one characteristic size (or column density). Evidently regions containing O VI-bearing material exist on both large and small scales, thus preventing a convergence to a nearly single number for the average density $n(\mathrm{O} \mathrm{VI})$ over great distances. This picture is not surprising, in the light of evidence gathered 
elsewhere that there are shells and superbubbles. Structures likely to contain hot gas are seen over many size scales (Heiles 1984).

\section{Interstellar Molecular Hydrogen}

Molecular hydrogen is the most abundant species in the interstellar medium, comprising most of the mass in dense clouds. Stars and planetary systems form from these clouds, and $\mathrm{H}_{2}$ also plays key roles in cloud chemistry.

Molecular hydrogen has numerous allowed ground-state electronic transitions in the FUSE bandpass $(\lambda<1130 \AA)$, but none in other wavelength regions. As a homonuclear diatomic molecule, $\mathrm{H}_{2}$ has no dipole moment, hence no allowed transitions in either the vibrational or rotational regimes. The easiest way to observe cold (ground state) $\mathrm{H}_{2}$ in the quiescent interstellar medium is through the Lyman (B-X) and Werner (C-X) electronic transitions, which lie between $844 \AA$ and $1108 \AA$. Hence FUSE is a fundamentally important tool for studies of molecular hydrogen.

\subsection{The FUSE Molecular Hydrogen Surveys}

Presented by T. P. Snow, CASA, University of Colorado. tsnow@casa.colorado.edu

The following subsections describe the scientific goals of the FUSE PI Team survey of molecular hydrogen, the results to date, as well as future prospects. The FUSE PI Team has divided studies of molecular hydrogen into two areas: (1) observations of $\mathrm{H}_{2}$ in diffuse interstellar environments; and (2) studies of $\mathrm{H}_{2}$ in translucent clouds. For the most part, the diffuse $\mathrm{H}_{2}$ program uses data being obtained for other PI-team programs, so only a few new targets are being observed specifically for $\mathrm{H}_{2}$. On the other hand, the translucent cloud $\mathrm{H}_{2}$ program requires all new observations, as heavily-reddened stars are normally avoided by observers not specifically interested in the interstellar medium, because dust extinction and spectral contamination by $\mathrm{H}_{2}$ bands are nuisances otherwise.

Most of the diffuse $\mathrm{H}_{2}$ targets are being observed for the warm gas, Galactic halo, and hot star winds programs being conducted by the FUSE PI team. Over 200 stars will eventually be included. The 35 stars specifically selected for the translucent cloud $\mathrm{H}_{2}$ survey were chosen on the basis of several criteria: substantial reddening; known UV dust extinction curve, sampling a variety of curve types; the existence of high-resolution optical interstellar line spectra, showing minimal velocity component structure; and early spectral type. Other supporting data, such as complete optical spectra, infrared magnitudes and spectra, ultra-high resolution optical line profiles, and high-resolution HST/STIS spectra of selected ions and molecules lying outside the FUSE spectral range, are also being obtained.

The main science goals of the diffuse $\mathrm{H}_{2}$ survey are: Measure $\mathrm{H}_{2}$ column densities in a variety of environments such as warm, ionized gas, high-velocity clouds, and the Galactic halo; determine cloud physical conditions from the analysis of rotational excitation in $\mathrm{H}_{2}$; survey the abundance of $\mathrm{H}_{2}$ relative to dust in environments with differing metallicities, such as the Magellanic Clouds; sample 
the $\mathrm{CO} / \mathrm{H}_{2}$ correlation; and determine $\mathrm{HD}$ column densities as a constraint on cosmic ray ionization.

The principal goals of the translucent cloud molecular hydrogen survey are: Determine $\mathrm{H}_{2}$ abundances, hence total cloud masses, for lines of sight with up to 5 magnitudes of visible extinction $\left(A_{V}\right)$; extend the $\mathrm{CO} / \mathrm{H}_{2}$ correlation to $A_{V}$ $\approx 5$; analyze cloud physical conditions from $\mathrm{H}_{2}$ rotational excitation measures; determine the $\mathrm{HD} / \mathrm{H}_{2}$ ratio as a potential indicator of atomic $\mathrm{D} / \mathrm{H}$; constrain the chemistry of translucent clouds; and study the relationships between molecular fraction and other cloud parameters such as extinction curve properties and depletions. The data from the translucent clouds survey will be used also in related studies such as determinations of dust extinction down to the Lyman limit, and of abundances and depletions of atomic species having transitions in the FUSE bandpass.

First Results of the $\mathrm{H}_{2}$ Surveys Perhaps the most striking result from the diffuse $\mathrm{H}_{2}$ survey is that molecular hydrogen is ubiquitous, with bands showing up in most spectra obtained to date. This includes extragalactic sources, which reveal $\mathrm{H}_{2}$ in our Galactic halo, as well as virtually every Galactic disk line of sight. A first paper on diffuse $\mathrm{H}_{2}$ was published by Shull et al. (2000b), and another is in press (Tumlinson et al. 2001). This second study focuses on the Magellanic Clouds, and suggests that the molecular fraction for hydrogen appears to be lower in low-metallicity environments than in the Milky Way. The incidence of $\mathrm{H}_{2}$ detections in the SMC is a bit greater than in the LMC, but this may be a selection effect, since the selected SMC targets have greater reddenings on average. Only an unbiased sample will reveal whether the SMC actually has a higher $\mathrm{H}_{2}$ to extinction ratio than the LMC. In addition to the Magellanic Clouds, there have been detections of $\mathrm{H}_{2}$ in high-velocity clouds, such as the Magellanic Stream (Sembach et al. 2001).

The first star observed for the translucent cloud $\mathrm{H}_{2}$ survey was $\mathrm{HD} 73882$, which has $E(B-V)=0.72$ and $A_{V}=2.44$. Results have been published in Snow et al. (2000). The analysis was done by a combination of profile fitting (for the damped $J=0$ and $J=1$ bands) and curve-of-growth analysis (for the saturated higher $J$-levels and HD) based on the line-of-sight velocity structure derived from ground-based optical interstellar line profiles obtained at very high spectral resolving power.

The $\mathrm{H}_{2}$ total column density for this line of sight is $N\left(\mathrm{H}_{2}\right)=1.2 \times 10^{21}$ $\mathrm{cm}^{-2}$, about three times larger than the values for diffuse clouds, such as the $\zeta$ Ophiuchi line of sight, that were previously measured in the far-UV. The molecular fraction in the HD 73882 line of sight is $f=2 N\left(\mathrm{H}_{2}\right) /[N(\mathrm{HI})+$ $\left.2 N\left(\mathrm{H}_{2}\right)\right]=0.65$, comparable to the value for $\zeta$ Oph. We detected absorption lines arising from rotationally excited states up to $J=7$, with the $N(J=1) / N(J=0)$ ratio indicating a gas kinetic temperature of $58 \mathrm{~K}$, while the $J \geq 2$ lines fit a rotational temperature of $307 \mathrm{~K}$. These results, too, are similar to those found previously for diffuse clouds such as the $\zeta$ Oph line of sight.

Subsequently some 16 of the translucent cloud $\mathrm{H}_{2}$ survey stars have been observed, and column densities and rotational excitations derived. The total $\mathrm{H}_{2}$ abundances show a rather small range, as do the kinetic temperatures and the molecular fractions. Evidently the small sample of translucent clouds observed so far are quite uniform in their properties. It remains to be seen whether all 
translucent clouds fit this pattern; if so, we might conclude that "translucent" cloud lines of sight in general are actually composites of several diffuse clouds, rather than representing a new physical and chemical regime. We hope to answer this question through our continued FUSE molecular hydrogen survey.

Future Plans The diffuse $\mathrm{H}_{2}$ survey will proceed with the analysis of molecular hydrogen in a variety of interstellar environments, with the selection of lines of sight still largely derived from observations for other FUSE programs. In order to expand the study of $\mathrm{H}_{2}$ in the Magellanic Clouds, FUSE Guest Investigator observing time was recently awarded to determine the $\mathrm{H}_{2}$ abundances toward several stars in the Clouds that were selected specifically to have enough extinction to be likely candidates for $\mathrm{H}_{2}$ detection. These observations will support more definitive explorations of questions such as the the ubiquity of molecular hydrogen in the Magellanic Clouds, the effect of metallicity on $\mathrm{H}_{2}$ formation, the relationship between $\mathrm{H}_{2}$ and dust extinction, and the $\mathrm{CO} / \mathrm{H}_{2}$ correlation.

The translucent cloud $\mathrm{H}_{2}$ team plans to complete the FUSE observations as well as other supporting observations at other wavelengths in order to develop a dataset that should have widespread utility in testing and exploring the science goals outlined above. We expect to make significant progress in determining the properties of translucent clouds, while also developing useful constraints on the chemistry and physics of the interstellar medium in general.

\section{2. $\mathrm{H}_{2}$ toward HD 34078}

\section{Poster presented by F. Lepetit, Observatoire de Paris-Meudon lepetit@murano.obspm.fr}

We give preliminary results of the analysis of a FUSE observation of HD 34078. $\mathrm{H}_{2}$ absorption is seen from highly excited levels (at least up to $\mathrm{v}=0, J$ $=10$, i.e. $8700 \mathrm{~K}$ above the ground level). The inferred total $\mathrm{H}_{2}$ column density is $\log \left[N\left(\mathrm{H}_{2}\right)\right] \simeq 20.81$.

The HD 34078 spectrum presented here is the first of a series aiming at a study of the small scale distribution of $\mathrm{H}_{2}$ in the interstellar medium, using a method similar to that employed by Marscher et al. (1993) for $\mathrm{H}_{2} \mathrm{CO}$. It has been obtained with FUSE in January 2000. HD 34078 is a O9.5 star extincted by a foreground molecular cloud $(E(B-V)=0.52)$. Many species have already been detected toward this direction: $\mathrm{H}_{\mathrm{I}}$ and $\mathrm{CO}$ column densities have been measured by Mc Lachlan and Nandy (1984) from an IUE spectrum while CH, $\mathrm{CN}, \mathrm{C}_{2}$ and $\mathrm{CH}^{+}$have been observed by Federman et al. (1994) and Allen(1994).

The $\mathrm{LiF} 1$ channel data have been used because of their higher signal to noise ratio. A portion of the spectrum is shown in Figure 5, around $1050 \AA$ where damped $\mathrm{H}_{2}$ lines are present.

The FUSE spectrum appears to be very rich: many atomic lines as well as molecular lines are detected from $\mathrm{HI}, \mathrm{ArI}, \mathrm{FeII}, \mathrm{CI}_{\mathrm{I}} \mathrm{CI}^{*}, \mathrm{CI}^{* *}$ or $\mathrm{H}_{2}, \mathrm{HD}$ and $\mathrm{CO}$. Here, we shall focus on $\mathrm{H}_{2}$ column densities as a function of excitation energy. The spectrum has been analysed using two different tools, a fitting procedure written by $M$. Lemoine (private communication) based on a $\chi^{2}$ minimization method and the LYMAN software which is part of the MIDAS package. 


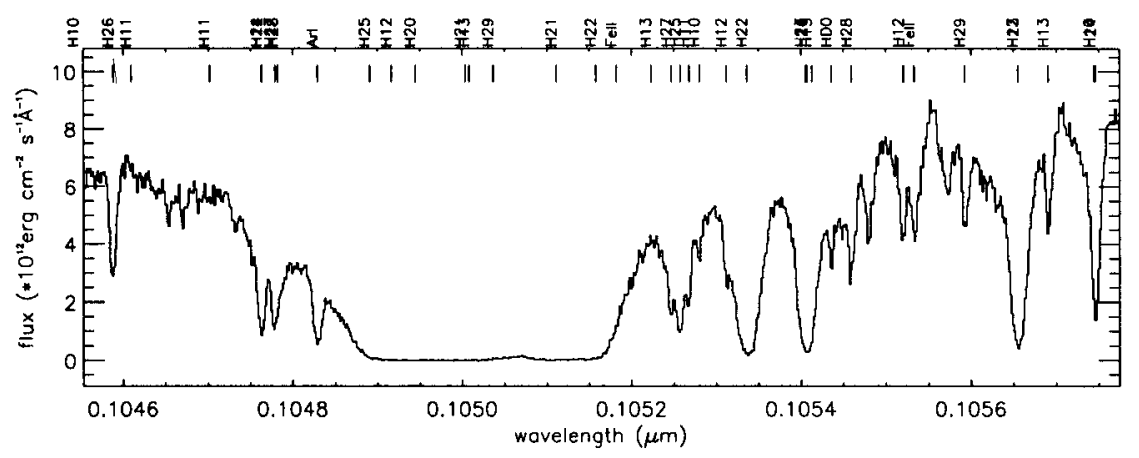

Figure 5. A portion of the FUSE spectrum of HD 34078. Nomenclature is $\mathrm{H} 2 J$ for $\mathrm{H}_{2}, \mathrm{v}=0, J$, where $J$ can take the value 0 to $9, \mathrm{H} 1 J$ for $\mathrm{H}_{2}, \mathrm{v}=1, J$, and $\mathrm{HD} J$ for $\mathrm{HD}, \mathrm{v}=0, J$. Transitions from highly excited levels of $\mathrm{H}_{2}$ as well as from the $\mathrm{v}=0, J=0$ level of HD are seen. Note that not all species are shown.

We adopt a Doppler $b$-value of $3 \mathrm{~km} \mathrm{~s}^{-1}$ for the first levels $(J=0,1,2,3)$ as suggested by $\mathrm{CH}$ observations (Allen 1994). This value may not be appropriate for high levels; a curve of growth analysis performed for the $J=7$ level yields $b=4 \mathrm{~km} \mathrm{~s}^{-1}$, indicating that if any, the variation of $b$ with excitation energy is not strong. For $J=4,5 \ldots$, we therefore assume $b=4 \mathrm{~km} \mathrm{~s}^{-1}$.

We clearly detect the Lyman $\beta$ line (although blended with $\mathrm{H}_{2}$ lines), from which we estimate a $\mathrm{HI}$ column density $\log \left[N\left(\mathrm{HI}_{\mathrm{I}}\right)\right]=21.40 \pm 0.05$, somewhat larger than that obtained from IUE (Mc Lachlan and Nandy 1984).

For the first time, molecular hydrogen absorption is observed from $\mathrm{v}=1$ levels (the highest with $J=5$, lying at $E=8400 \mathrm{~K}$ above the ground state; for absorption from $\mathrm{v}=3$ lines, see Federman et al. 1995). Weak features from $\mathrm{v}$ $=0, J=10(8700 \mathrm{~K})$ are detected. Lines from still higher levels have not been systematically searched for yet and may be present. Column densities for the eight first levels are given in the table (with their $3 \sigma$ uncertainty). Up to $\mathrm{v}=0$, $J=3$, the $N$ value does not depend on the adopted $b$; this is no longer true for higher levels.

\begin{tabular}{|c|c|c|c|c|c|c|c|}
\hline level & $\left.\log \left[\mathrm{N}_{(\mathrm{H}}, J\right)\right]$ & & level & $\log \left[\mathrm{N}\left(\mathrm{H}_{2}, J\right)\right]$ & & level & $\log \left[\mathrm{N}\left(\mathrm{H}_{2}, J\right)\right]$ \\
\hline$J=0$ & $20.50 \pm 0.05$ & & $J=3$ & $18.79 \pm 0.06$ & & $J=6$ & $16.06 \pm 0.20$ \\
$J=1$ & $20.50 \pm 0.05$ & & $J=4$ & $17.67 \pm 0.09$ & & $J=7$ & $15.59 \pm 0.11$ \\
$J=2$ & $19.18 \pm 0.05$ & & $J=5$ & $17.32 \pm 0.15$ & & & \\
\hline
\end{tabular}

The total $\mathrm{H}_{2}$ column density towards HD 34078 is $6.4 \times 10^{20} \mathrm{~cm}^{-2}$. This line of sight appears remarkable in the distribution of that $\mathrm{H}_{2}$ gas over the 17 levels from which we detect absorption. We are currently extending our models to determine which constraints can be obtained from the data on the processes responsible for the high excitation observed.

A more detailed analysis of the spectrum, including in particular HD and $\mathrm{CI}$ lines from which the $\mathrm{HD} / \mathrm{H}_{2}$ and gas density can be determined, will be published elsewhere. 


\title{
7. Studies of the Intergalactic Medium
}

\author{
Presented by J. M. Shull, CASA, University of Colorado. \\ mshull@casa.colorado.edu
}

With its sensitivity and spectral resolution in the far ultraviolet, FUSE provides an outstanding means of observing the intergalactic medium (IGM). In this section, we describe FUSE observations in three areas of IGM research: (1) the Lyman- $\beta$ forest; (2) detections of heavy elements in the IGM; and (3) measurements of He II absorption at redshifts $2<z<3$.

\subsection{The Lyman- $\beta$ Forest}

As part of the Early Release Observations (ERO), we performed a moderateresolution (20-25 $\mathrm{km} \mathrm{s}^{-1}$ ) FUSE study of the low-redshift IGM. The resulting paper (Shull et al. 2000a) reported on studies of 7 extragalactic sightlines and 12 Ly $\beta$ absorbers that correspond to Ly $\alpha$ lines detected by HST/GHRS and STIS. These absorbers appear to contain a significant fraction of the low- $z$ baryons and were a major discovery of the HST spectrographs. Using FUSE data, with $40 \mathrm{~m} \AA$ $(4 \sigma) \operatorname{Ly} \beta$ detection limits, we employed the equivalent width ratio of $\operatorname{Ly} \beta / \operatorname{Ly} \alpha$ and occasionally higher Lyman lines, to determine the Doppler parameter, $b$, and accurate column densities, $N(\mathrm{HI})$, for moderately saturated lines. We detected Ly $\beta$ absorption corresponding to all Ly $\alpha$ lines with equivalent widths $W_{\lambda} \geq 200$ $\mathrm{m} \AA$.

The $\operatorname{Ly} \beta /$ Ly $\alpha$ ratios yield a preliminary distribution function of Doppler parameters, with mean $\langle b\rangle=31.4 \pm 7.4 \mathrm{~km} \mathrm{~s}^{-1}$ and median $28 \mathrm{~km} \mathrm{~s}^{-1}$, comparable to values measured by the Keck telescope at redshifts $z=2.0-2.5$. If thermal, these $b$-values correspond to $T_{\mathrm{HI}} \approx 50,000 \mathrm{~K}$, although the inferred Doppler parameters are considerably less than the widths derived from Ly $\alpha$ profile fitting, $\left\langle b / b_{\text {width }}\right\rangle=0.52$. The typical increase in column density over that derived from profile fitting is $\Delta \log N(\mathrm{H} \mathrm{I})=0.3$ but ranges up to 1.0 dex. Our data suggest that the low- $z$ Ly $\alpha$ absorbers contain sizable non-thermal motions or velocity components in the line profile, perhaps arising from cosmological expansion and infall.

Since the ERO paper, we have accumulated further FUSE data on the IGM toward extragalactic targets. Two strong $\mathrm{Ly} \alpha$ absorbers are seen in Ly $\beta$ toward $3 \mathrm{C} 273$ at $c z=1015$ and $1586 \mathrm{~km} \mathrm{~s}^{-1}$. The $1586 \mathrm{~km} \mathrm{~s}^{-1}$ absorber is surprisingly strong, and is also seen in six other lines in the Lyman series, including $\operatorname{Ly} \gamma, \operatorname{Ly} \delta, \operatorname{Ly} \epsilon, \operatorname{Ly} \zeta, \operatorname{Ly} \eta$, and $\operatorname{Ly} \theta$. The concordance of equivalent widths for the seven Lyman lines in this absorber yields a column density in the range log $N(\mathrm{H} \mathrm{I})=15.5_{-0.3}^{+0.4}$ and $b=20 \pm 3 \mathrm{~km} \mathrm{~s}^{-1}$. Similar measurements have been done for strong absorbers toward PG $1211+143$ at $c z=15,300 \mathrm{~km} \mathrm{~s}^{-1}$ and 19,400 $\mathrm{km} \mathrm{s}^{-1}$. These Ly $\beta / \mathrm{Ly} \alpha$ concordance determinations are critical for making accurate determinations of $N_{\mathrm{HI}}$, which plays an important role in the ionization corrections for heavy elements (for example, C IV $/ \mathrm{H} \mathrm{I}, \mathrm{C}$ III/H I, Si III/H I, $\mathrm{O} \mathrm{VI} / \mathrm{H} \mathrm{I}$ ) needed for accurate metallicities in these absorbers. Ultimately, with a large database of $\operatorname{Ly} \beta$ measurements, we intend to measure an accurate distribution of $\mathrm{H}$ I column densities in the low-redshift Ly $\alpha$ forest (see Penton, Shull, \& Stocke 2000). 


\subsection{Metals in the Hot Intergalactic Gas}

FUSE is sensitive to resonance lines of several heavy elements expected in the interstellar and intergalactic medium. These lines include the $\mathrm{O}$ VI doublet $(1031.926,1037.617 \AA), \mathrm{C}$ III $(977.020 \AA), \mathrm{N}$ III $(989.799 \AA)$, and numerous farUV lines of $\mathrm{CII}, \mathrm{Fe} I \mathrm{I}, \mathrm{O}$, and NI.

The O VI doublet has been observed by HST in several IGM absorbers at redshifts $z>0.14$ (Tripp et al. 2000; Tripp \& Savage 2000). With an assumed metallicity of $1 / 10$ solar and a conservative assumption that the fraction of oxygen in the O VI ionization stage is 0.2 , these authors obtain a value for the baryon density in this hot gas, $\Omega_{b} \geq 0.004 h_{75}^{-1}$. This is comparable to the combined cosmological mass density of stars and cool gas in galaxies and X-rayemitting gas in galaxy clusters at low redshift.

Similar high values for the baryons have been estimated for the reservoir of $\mathrm{H}^{+}$and $\mathrm{H}^{\circ}$ in the photoionized Ly $\alpha$ forest (Penton, Shull, \& Stocke 2000). It appears likely that the OVI absorbers are associated with the $\mathrm{HI}$ ( Ly $\alpha$, $\operatorname{Ly} \beta$ ) absorbers, although it is not certain whether the O vI co-exists in the same photoionized gas as the Ly $\alpha$ absorbers. Owing to its high ionization production threshold $(113.87 \mathrm{eV}), \mathrm{OVI}$ is usually collisionally ionized in gas at $T \approx 10^{5.7 \pm 0.3} \mathrm{~K}$. Thus, one must be careful not to double-count the $\mathrm{O}$ VI and Ly $\alpha$ absorbers in the statistics of $d N / d z$ used to infer $\Omega_{b}$.

With FUSE, we now have the opportunity to study both OVI and Ly $\beta$ absorption for the low-redshift IGM $(0<z<0.14)$. We have already measured strong $\mathrm{O}$ VI in low- $z$ absorbers toward PG0853+414 $(z=0.06805)$ and PG1211+143 $\left(c z=15,300 \mathrm{~km} \mathrm{~s}^{-1}\right.$ and $\left.19,400 \mathrm{~km} \mathrm{~s}^{-1}\right)$. The $\mathrm{C}$ III $\lambda 977$ line is also seen towards the last two absorbers. With both C III $\lambda 977$ and C IV $\lambda 1548$ (from HST), we have measurements of ionization states from a common element. This provides an opportunity to infer the photoionization conditions, which in turn yield more accurate metallicities. Although the current statistics are still small, the FUSE survey of the low-redshift IGM has the promise to measure metallicities for both the photoionized and collisionally ionized components of the IGM at $z<0.14$.

\subsection{He II Absorption}

One of the major goals of the FUSE IGM studies is to measure the redshifted absorption of the He II $\lambda 303.78 \AA$ resonance line in the IGM toward high- $z$ QSO. Although the FUSE bandpass allows us to detect He II at redshifts $2.05<$ $z<2.90$, this is an exceedingly difficult task, because there are few UV-bright QSO at these redshifts. As a result, the He II measurements require very long exposures of $V=15.5-16.5$ QSO, with signals near the level of the FUSE background.

This He II absorption, often referred to as the "He II Gunn-Peterson Effect", has been detected previously by HST toward four QSO: HS1700+64169 $\left(z_{\mathrm{em}}=\right.$ 2.74), Q0302-003 $\left(z_{\mathrm{em}}=3.29\right)$, Q1935-69 $\left(z_{\mathrm{em}}=3.18\right)$, and HE2347-4342 $\left(z_{\mathrm{em}}=\right.$ 2.885). Theoretical models (Fardal, Giroux, \& Shull 1998) suggest that the He II absorption arises primarily from HeII in the Ly $\alpha$ forest clouds, photoionized by a radiation field produced by AGN and starburst galaxies. A comparison to the H I Ly $\alpha$ absorbers indicates that the radiation field must be "soft" (relatively few photons in the 4 Rydberg He Il continuum) so that $N(\mathrm{He} \mathrm{II}) / N(\mathrm{HI})$ is large, 
perhaps $\geq 100$. The radiative transfer studies further suggest that the mean He II optical depth, $\tau_{\mathrm{He} \text { II }}$, should decrease substantially at $z \leq 3$, with significant fluctuations arising from small-number statistics in the absorber population and fluctuations in the number of ionizing sources within the " $\tau_{H}=1$ visibility horizon".

With FUSE, we have confirmed the He II absorption toward HS1700+6416 (between 1090 and $1138 \AA$ ). More recently, we have measured He II absorption in a far-ultraviolet spectrum of HE2347-4342, covering the 912-1187 $\AA$ wavelength range. Our observations comprise nearly $\sim 400 \mathrm{ks}$ of data obtained during orbital night. Binned to a resolution of $0.1 \AA$, the data have a signal-to-noise ratio of $\sim 5$, and they are adequate to resolve individual absorption features and voids in the He II Ly $\alpha$ forest. Based on a direct comparison of features resolved in both this spectrum and a Keck spectrum of the H I Ly $\alpha$ forest, we will be able to constrain the ionization state of the IGM and the spectral shape of the metagalactic ionizing flux. We will also obtain information about the He II opacity of the IGM and its fluctuations over the redshift interval $z=2.1-2.8$, by binning the spectrum to optimize the signal-to-noise ratio.

\section{FUSE Observations of Active Galactic Nuclei}

\section{Presented by G. Kriss, Space Telescope Science Institute. gak@stsci.edu}

The FUSE PI-team program directed toward the study of AGN piggy-backs off data acquired as part of the larger PI-team study of O VI absorption in the Galactic halo and the study of the deuterium to hydrogen ratio along different sight lines through the Galactic halo and high-velocity clouds. As part of these programs, the $\sim 100$ UV-brightest AGN are being surveyed at low to moderate $\mathrm{S} / \mathrm{N}$ ratios to determine the best sight lines for further study. A few intrinsically interesting AGN are being observed at high $\mathrm{S} / \mathrm{N}$ as part of the "medium-size" team project as are a few Seyfert 2 galaxies. In addition to the FUSE observations, an HST snapshot program is obtaining contemporaneous spectra at longer UV wavelengths using the STIS G140L and G230L gratings, and current-epoch ground-based optical spectra covering $\sim 3500-9000 \AA$ are being obtained by $R$. Green and M. Brotherton at KPNO. The primary scientific goals of the FUSE AGN program are to (1) determine the far-UV continuum shape of low-redshift AGN; (2) examine the strength and profile of the broad O VI emission line; (3) search for intrinsic $\mathrm{O}$ VI and Lyman-limit absorbers and study their kinematics; (4) search for intrinsic $\mathrm{H}_{2}$ absorption that may be associated with the obscuring torus; and (5) study the strengths of other far-UV resonance lines such as CIII $\lambda 977$ and N III $\lambda 991$.

As of 2000 July 1, 47 AGN at $z<1$ had been observed by FUSE. Forty six of these are Type 1 AGN; one is the Seyfert 2 Mrk 463, which was not detected. Of the 28 Type 1 s with $z<0.15$ (so that O vi falls in the FUSE bandpass), strong, broad O VI emission is visible in all but one (PKS $0558-504$ ). Four of the 28 also show strong narrow O VI emission lines. Approximately 40\% (11 of 28) show intrinsic O VI absorption. No intrinsic Lyman limits are detected, and no intrinsic molecular hydrogen absorption is visible. Representative spectral energy distributions for four of the AGN in this sample are shown in Figure 6. 


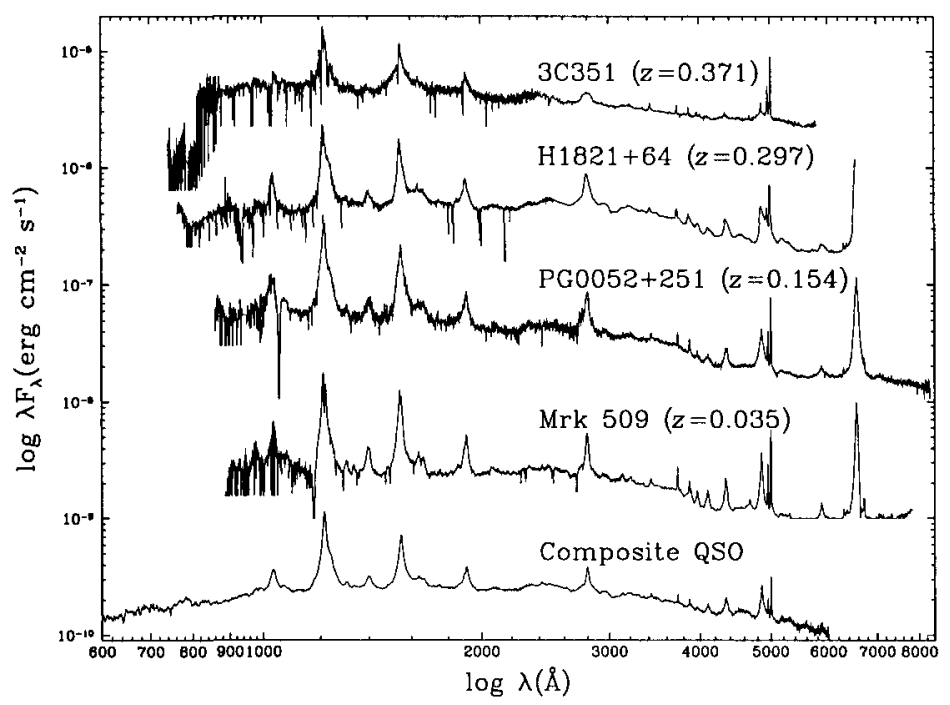

Figure 6. Combined FUSE , HST, and ground-based data for four AGN show rest-frame spectral energy distributions peaking at $\sim 1000$ $\AA$, in qualitative similarity to the composite QSO spectrum of Zheng et al. (1997). The sharp drop at the short wavelength end of the 3C 351 spectrum is a foreground Lyman-limit system at $z=0.22$.

Note the overall qualitative similarity of these AGN spectra to the Zheng et al. (1997) composite. While it is too early to draw definitive conclusions, this suggests that there is little evolution in mean spectral shape from redshifts of $\sim 1$, as represented by the composite, to the current epoch.

\subsection{FUSE Observations of Warm Absorbers}

The "warm" (or, ionized) absorbing gas that is common in the X-ray spectra of low-redshift AGN is a major new component of their near-nuclear structure. About half of all low-redshift AGN show absorption by ionized gas (Reynolds 1997, George et al. 1998), and a similar fraction show associated UV absorption in highly ionized species such as CIV (Crenshaw et al. 1999). For AGN that have been observed at moderate to high spectral resolution in both the X-ray and the UV, there is a one-to-one correspondence between objects showing $\mathrm{X}$ ray and UV absorption, suggesting that the phenomena are related in some way (Crenshaw et al. 1999). The gas has a total mass exceeding $\sim 10^{3} M_{\odot}$ (greater than the broad-line region, or BLR), and is outflowing at a rate $>0.1 M_{\odot} \mathrm{yr}^{-1}$ (10x the accretion rate in some objects, Reynolds et al. 1997).

A key question for understanding warm absorbers is how the X-ray and UV spectral domains are related. In some cases, UV absorbing gas may be directly associated with the X-ray warm absorber (3C351: Mathur et al. 1994; NGC 5548: Mathur et al. 1995). In other cases, however, the UV gas appears to be in an even lower ionization state, and there is no direct relation between the 


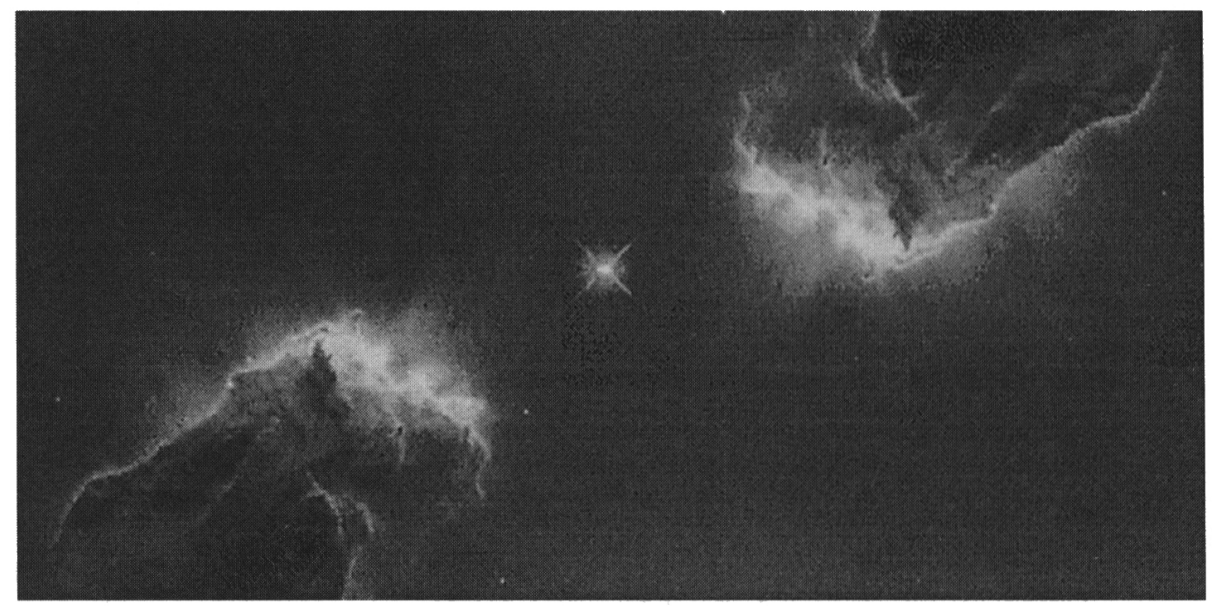

Figure 7. Artistic rendering of how a molecular torus surrounding an AGN might appear based on HST images of the Eagle Nebula.

X-ray absorption and the UV absorption (NGC 4151: Kriss et al. 1995; NGC 3516: Kriss et al. 1996a, 1996b; NGC 7469: Kriss et al. 2000a). High spectral resolution observations of O VI absorption in AGN with X-ray warm absorbers is proving to be an invaluable tool for definitively analyzing these issues.

The disparity between the UV and X-ray absorbers is illustrated most clearly by FUSE observations of the Seyfert 1 galaxy Mrk 509 (Kriss et al. $2000 \mathrm{~b}$ ). This observation resolves the UV absorption into seven kinematic components. In addition to the $\mathrm{O}$ VI and $\operatorname{Ly} \beta$ absorption shown here, $\mathrm{C}_{\text {III }} \lambda 977$ lines are seen in the lowest-ionization components, and Lyman-line absorption up to Ly $\zeta$ permits an accurate measure of the neutral hydrogen column density and its covering fraction. Comparison of the $\mathrm{O}$ VI, $\mathrm{HI}$, and $\mathrm{C}$ III column densities to photoionization models permits one to independently assess the total column density of each kinematic component. Of the seven systems present, only one near the systemic velocity of Mrk 509 stands out clearly as having a level of ionization and total column density compatible with an X-ray warm absorber. While the total equivalent width of the UV absorption is dominated by the other six components, the X-ray absorption that would be associated with them is negligible. It is puzzling, however, that the high-ionization component lies so close to the systemic velocity. Most absorbers in other AGN (including most of the other components in this one) are blue-shifted relative to the host galaxy, and this is true for the high-resolution X-ray spectra obtained so far with Chandra: the X-ray absorption lines in NGC 5548 are blue-shifted by $\sim 280 \mathrm{~km} \mathrm{~s}^{-1}$ (Kaastra et al. 2000) and by $\sim 440 \mathrm{~km} \mathrm{~s}^{-1}$ in NGC 3783 (Kaspi et al. 2000). Clearly a larger sample of objects that would permit us to assess these differences is essential.

The multiple kinematic components frequently seen in the UV absorption spectra of AGN clearly show that the absorbing medium is complex, with separate UV and X-ray dominant zones. One potential geometry is high density, 
low column UV-absorbing clouds embedded in a low density, high ionization medium that dominates the X-ray absorption. This is possibly a wind driven off the obscuring torus or the accretion disk. What would this look like in reality? Detailed modeling of dense molecular gas irradiated by the ionizing continuum of an AGN is likely to miss the complexities that arise as material is ablated from the surface and flows away. We will not soon get a close-up look at this aspect of an AGN, so it is instructive to look at nearby analogies. The HST images of the pillars of gas in the Eagle Nebula, M16, show the wealth of detailed structure in gas evaporated from a molecular cloud by the UV radiation of nearby newly formed stars (Hester et al. 1996). Figure 7 shows what this might look like in an AGN - a dense molecular torus surrounded by blobs, wisps, and filaments of gas at various densities. It is plausible that the multiple UV absorption lines seen in AGN with warm absorbers are caused by high-density blobs of gas embedded in a hotter, more tenuous, surrounding medium, which is itself responsible for the $\mathrm{X}$-ray absorption. Higher density blobs would have lower ionization parameters, and their small size would account for the low overall column densities.

At sight lines close to the surface of the obscuring torus, one might expect to see some absorption due to molecular hydrogen. Given the dominance of Type $1 \mathrm{AGN}$ in our observations so far, the lack of any intrinsic $\mathrm{H}_{2}$ absorption is not too surprising since our sight lines are probably far above the obscuring torus. NGC 4151 and NGC 3516 are examples where the inclination may be more favorable since these objects have shown optically thick Lyman limits in the past (Kriss et al. 1992, 1995, 1996a), but our FUSE observations do not show such high levels of neutral hydrogen at the current epoch. Molecular hydrogen will not survive long in an environment with a strong UV flux. Given this, one might think that any sightline in an AGN in which a strong UV continuum was visible could not show $\mathrm{H}_{2}$ absorption since the $\mathrm{H}_{2}$ would not be optically thick enough to be self shielding. However, if the $\mathrm{H}_{2}$ is contained in small, dense blobs that have ablated from the obscuring torus, these blobs could be thick enough to shield their cores and small enough that they do not completely obscure the central radiation source. Thus, the $\mathrm{H}_{2}$ absorption, if present, is likely to show up as optically thick, high-column-density components that only partially cover the continuum or broad lines. Our best prospects for seeing such material are in upcoming FUSE observations of NGC 1068.

In conclusion, FUSE offers numerous advantages for the study of absorbing gas in AGN. First, since the Ly $\alpha$ absorption in the HST bandpass is often saturated, it is difficult to deblend the various velocity components and obtain accurate HI column densities. The unsaturated high-order Lyman lines in the FUSE bandpass make accurate measurement of the $\mathrm{H}_{\mathrm{I}}$ column density straightforward. Second, the O VI resonance lines are a key link between the lower-ionization species seen in the UV and the higher-ionization gas observed with Chandra. The presence of different, adjacent ionization states in the two bandpasses makes it possible to ascertain the ionization structure in a more model-independent way. One need not make assumptions about abundances or use photoionization models, for example, as would be required in comparing $\mathrm{C}$ IV in the HST band to O VII and O VIII in the Chandra spectrum. Similarly, the ionization state of the UV-absorbing gas can be obtained more directly without recourse to assumptions about abundances by comparing the $\mathrm{C}$ III opacity in the FUSE bandpass at $977 \AA$ to C IV as observed with HST. Finally, after using O VI 
to identify the kinematic components in the absorbing gas associated with the X-ray warm absorber, the FUSE observations can be used to trace the kinematics of the X-ray absorbing gas at higher velocity resolution than is possible with the Chandra grating observations.

\section{Lyman Continuum Radiation from Galaxies}

Presented by J-M Deharveng, Laboratoire Astronomie Spatiale, Marseille. Jean-Michel.Deharveng@astrsp-mrs.fr

\subsection{Introduction}

The physical conditions in the intergalactic medium (IGM) and its constituents are known to be shaped by the ultraviolet ionizing background radiation. The intensity of this radiation field can be measured by its effects on the detectable gas, such as for instance the sharp edges of $\mathrm{H} \mathrm{I}$ emission in disks of galaxies or the proximity effect (e.g. Bechtold 1993, Shull et al. 1999 and references therein). Another approach is possible by summing up the contributions of QSOs and star-forming galaxies while accounting for the opacity of neutral hydrogen clouds in the IGM (e.g. Bechtold et al. 1987, Haardt \& Madau 1996 and references therein). In contrast to quasars, there are very scarce data in the EUV for galaxies. This situation can be partially circumvented by evolutionary synthesis models, arguments based on the rate of chemical enrichment in the universe (Songaila et al. 1990, Madau \& Shull 1996) or simply extrapolation from the $\mathrm{UV}$ or $\mathrm{H} \alpha$ luminosity density. In all cases, however, a major uncertainty remains as to the fraction of the EUV stellar radiation escaping neutral hydrogen (and dust) absorption in the parent galaxy and effectively released into the IGM.

The Lyman continuum $\left(\mathrm{Ly}_{c}\right)$ escape fraction is therefore a crucial parameter to measure in galaxies. Leitherer et al. (1995) have obtained upper limits on this parameter in four nearby galaxies with the Hopkins Ultraviolet Telescope (HUT). The wavelength range of FUSE, down to $905 \AA$, offers again the possibility to observe a few star-forming galaxies with their $L y_{c}$ radiation redshifted above the limit of H I photoelectric opacity in our Galaxy. The redshifts of these objects have to be large enough to get rid, in combination with the spectral resolution of FUSE, of the (Lyman series) absorption lines from neutral hydrogen in our Galaxy as emphasized by Hurwitz et al. (1997). The spectral resolution of FUSE is also expected to allow correction for molecular hydrogen absorption in our Galaxy. We report here the observation of Mrk $54(z=0.0447)$.

\subsection{Observation of Mrk 54 and analysis}

Mrk 54 has been observed for a total duration of 27502 seconds. The spectrum shows the continuum flux declining towards short wavelengths and dropping to zero slightly before the redshifted Lyman break $(952.6 \AA)$ because of the accumulation of Lyman series absorption lines in the object itself. We have concentrated on the determination of an upper limit to the redshifted $\mathrm{Ly}_{c}$ flux. Essentially, we have defined a spectral window of $16 \AA$ as free as possible from airglow emission lines and Lyman series absorption lines by neutral hydrogen in 
the Galaxy. We have calculated the net count rate in this window resulting from the subtraction of the background measured in adjacent regions of the detector. The flux upper limit was defined as three times the dispersion of this net count rate. This upper limit was corrected for foreground dust extinction but not for absorption by molecular hydrogen in the Galaxy since there is no evidence for such features in the brightest part of the spectrum. The resulting upper limit on the flux of Mrk 54 at $\sim 900 \AA$ is $6.15 \times 10^{-16} \mathrm{erg} \mathrm{cm}^{-2} \mathrm{~s}^{-1} \AA^{-1}$ and by comparison with the $\mathrm{H} \alpha$ flux of $2.6 \times 10^{-13} \mathrm{erg} \mathrm{cm}^{-2} \mathrm{~s}^{-1}$ translates into a $\mathrm{Ly}_{c}$ escape fraction upper limit of $6.2 \%$.

\subsection{Results}

The $\mathrm{Ly}_{c}$ escape fraction upper limit of $6.2 \%$ in Mrk 54 is consistent with current estimates in the range $2 \%-10 \%$ as obtained from theoretical models (e.g. Dove et al. 2000) or indirect measurements (Tumlinson et al. 1999).

The present observation of Mrk 54 adds another significant limit to three other cases with upper limits of $3.2 \%, 5.2 \%$ and $11 \%$, as resulting from the observations of Leitherer et al. re-analyzed by Hurwitz et al. (1997).

Implications of the $\mathrm{Ly}_{c}$ escape fraction on the contribution of galaxies to the ionizing background have been addressed by a number of authors. Among others, Giallongo et al. (1997) and Shull et al. (1999) have shown that, at low redshifts, the galaxy contribution rivals that of QSOs for an escape fraction of $5 \%$. At high redshifts it is the recent detection of the $\mathrm{Ly}_{c}$ radiation in a composite spectrum of Lyman break galaxies (Steidel et al. 2000) that has now the most direct bearing on the role of galaxies in the re-ionization.

\section{Cool Stars}

Presented by A. K. Dupree, Harvard-Smithsonian Center for Astrophysics. dupree@cfa.harvard.edu

The outer atmospheres of cool stars radiate profusely in the far ultraviolet region, and the emission detected by FUSE formed in collisionally excited plasmas generally originates from regions spanning more than a decade in temperature. In particular, these temperatures from $10^{4} \mathrm{~K}$ to $3 \times 10^{5} \mathrm{~K}$ mark a critical region in a cool star atmosphere at the base of the transition region where the radiative losses reach a maximum and where, from study of the Sun, the acceleration in coronal holes becomes apparent and the atmospheric structure changes. Such differences are believed to signal the presence of changing conditions in the energy balance and dynamics of a stellar atmosphere.

The spectral resolution of FUSE allows measurement of line profiles that indicate mass flow, mass loss or accretion processes in these stars. The "timetag" mode of FUSE enables documentation of changes in line profiles during transient events. A powerful capability of far ultraviolet spectra resides in the presence of many lines whose ratios are sensitive to the local electron density: C III, Nevi, S II, S III, and S IV among them. These diagnostics can probe densities from $10^{7}$ to $10^{12} \mathrm{~cm}^{-3}$.

Several cool stars were observed during the Science Verification and Calibration phases of FUSE, others form a part of the research program of the 
Co-Investigator team. The four objects discussed below, AB Doradus, AG Draconis, AB Aurigae, and Beta Draconis, represent different evolutionary phases and well illustrate the capabilities of FUSE.
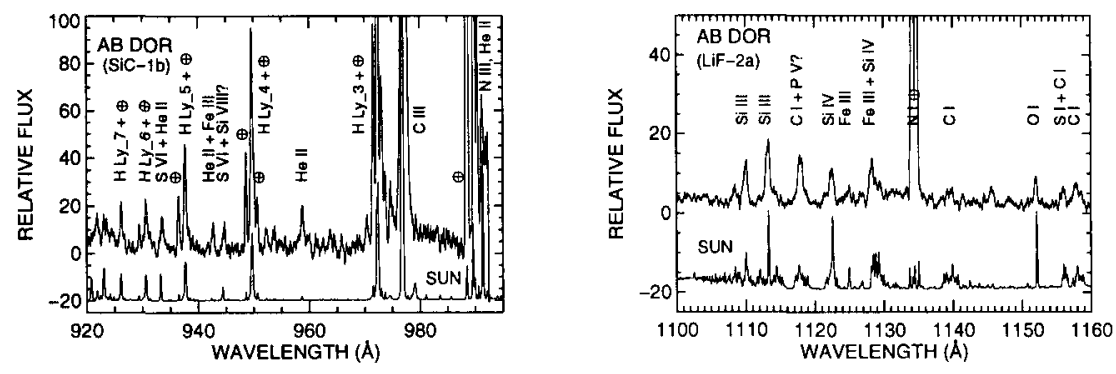

Figure 8. Sections of the AB Dor spectrum showing the Lyman series (observed at satellite night confirming their stellar origin) and emission from CIII, HeII, and S VI. Lines with contributions from airglow are marked by $\oplus$. The solar spectrum from SUMER/SOHO (below) shows some of the same transitions as AB Dor.
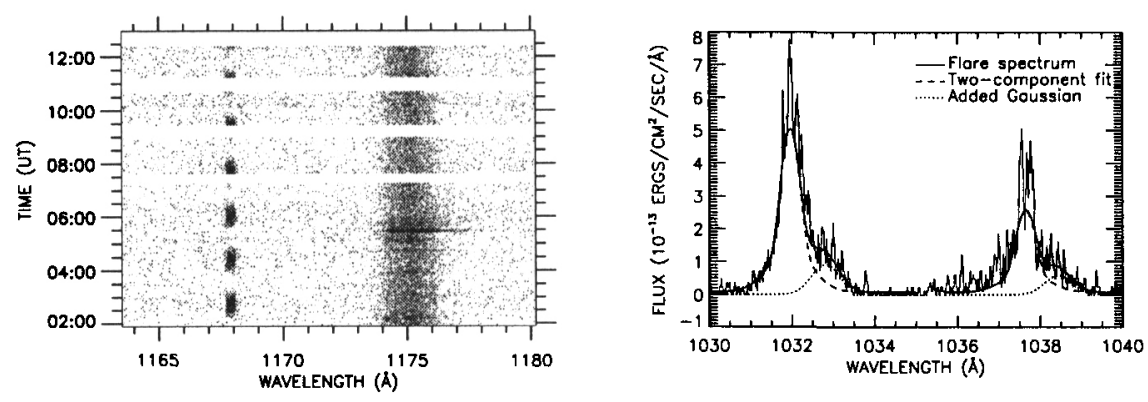

Figure 9. Left: Dynamic spectra of $\mathrm{C}$ III $\lambda 1176$ during the 1999 October 22 observation. Precursor flares occurred before the bright event at 05:28 UT where the flux increases and is shifted to longer wavelengths. The SAA causes gaps in the data; diurnal variation in the $\lambda 1168$ emission arises from second order scattered HeI ( $\lambda 584)$ airglow emission. Right: O VI profiles during the flare event showing a 2-Gaussian component fit to each O VI line, and the extra red-shifted component that appears during the flare.

\subsection{AB Doradus: a young active star}

AB Doradus (HD 36505) is a nearby (15 pc) rapidly rotating $\left(\mathrm{P}_{\text {rot }}=12.4 \mathrm{hr}\right.$ ), pre-main sequence star that exhibits high activity and frequent flaring (Collier Cameron et al. 1999). Early Release Observations described in Ake et al. (2000), and sections of the spectrum shown in Figure 8 shows the wide range of ions that 
are present, and the obviously broad lines as compared to the solar spectrum reflecting its $v \sin i$ of $90 \mathrm{~km} \mathrm{~s}^{-1}$.

We were fortunate to catch a flare on AB Dor (Fig. 9) and the photon counting mode of FUSE makes possible time resolution of line profiles and line fluxes. The brightening of the $\mathrm{C}$ III line was accompanied by a downflow shown both in $\mathrm{C}_{\mathrm{III}}$ and in higher temperature plasma where $\mathrm{O}$ VI is formed. Emission profiles of $\mathrm{O}$ VI show downflows centered at $+245 \mathrm{~km} \mathrm{~s}^{-1}$ and extending to +600 $\mathrm{km} \mathrm{s}^{-1}$ during this flaring episode. Whereas a flare on $\mathrm{AB}$ Dor observed in C IV exhibited blue-shifted emission (Vilhu et al. 1998), flares on AD Leo have shown substantial redshifts (Bookbinder, Walter \& Brown 1992). Downward-flowing flare material is a frequent occurrence in solar postflare magnetic loops and could explain the profiles observed here.

AB Dor has well known optical and ultraviolet light variations over its 12 $\mathrm{hr}$ rotational period, as active regions pass across the stellar disk. The time-tag mode of FUSE allows the spectra to be binned in specified time intervals (see Fig. 9), and rotational modulation is obvious in the transition region lines of $\mathrm{C}$ III and OVI. A maximum in the ultraviolet flux corresponds roughly to a minimum in the optical flux - as expected when dark spots are present on the photosphere.

\subsection{AG Draconis: a symbiotic system}

AG Dra (SAO 16931) is a symbiotic binary system consisting of a bright $\mathrm{K}$ giant and a hotter compact companion orbiting with a period of 554 days. The stellar wind from the $\mathrm{K}$ giant is deflected into an accretion disk or bright nebula by the compact object. High energy photons are produced by the white dwarf photosphere and by accretion. The high energy photons photoionize the gas surrounding the binary system. All of these components create a rich far ultraviolet spectrum that can probe the physical conditions and dynamics of material associated with the stars and near the system.

FUSE spectra (Young et al. 2000) were obtained of AG Dra as a FUSE Science Verification target. As might be expected from such a system, the spectrum (see Fig. 10) contains a strong ultraviolet continuum, emission lines of Ne v], Ne VI], S IV, S VI, O VI, C III, and the He II Balmer series. The O VI line exhibits a $P$ Cygni profile with absorption extending to $-200 \mathrm{~km} \mathrm{~s}^{-1}$. Because the observations were made at a phase when the compact object was positioned in front of the $\mathrm{K}$ giant, the $\mathrm{O}$ VI absorption of the hot continuum samples the dynamics of the material along the line of sight. Density diagnostics of $\mathrm{NeVr}$ ] suggest a nebula density between $10^{7}$ and $10^{10} \mathrm{~cm}^{-3}$.

\subsection{AB Aurigae}

AB Aur (HD 31293; A0pe) is a pre-mainsequence star classified as a Herbig Ae object, whose current state forms an important link for star formation. It marks the start of an evolutionary phase in which pre-mainsequence stars lose their near-IR excess and can be used to establish the age when planet signatures become visible in circumstellar disks. R-band images of the region around $\mathrm{AB}$ Aur show an extended reflection nebula, and STIS imaging $(570 \mathrm{~nm})$ reveals a nebula and associated structured disk as well as extended circumstellar nebulosity (Grady et al. 1999). 


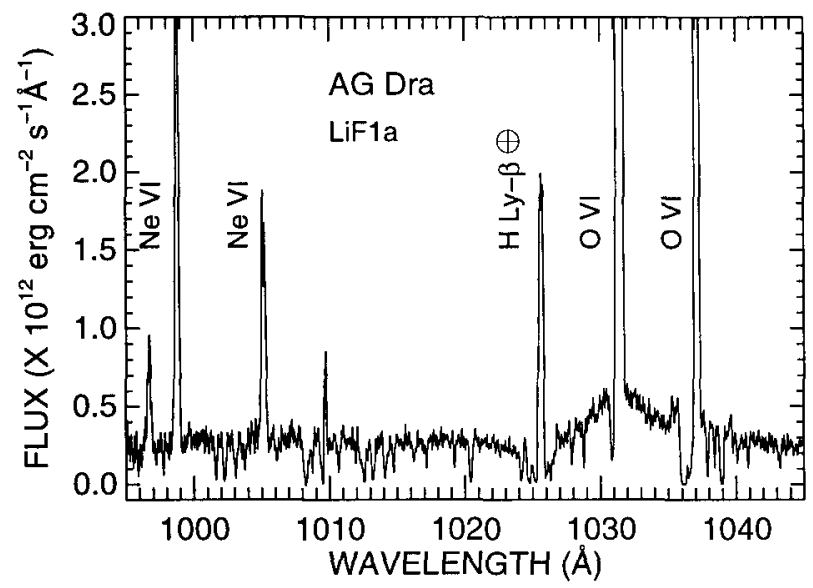

Figure 10. FUSE (LiF1a) spectrum of AG Dra showing the strong $\mathrm{O}$ VI (truncated profiles) and Ne VI emission. An enhanced continuum is apparent around the $\mathrm{OVI} \lambda 1032$ line most probably resulting from electron scattering of $\mathrm{O} V \mathrm{VI}$ in the surrounding material.

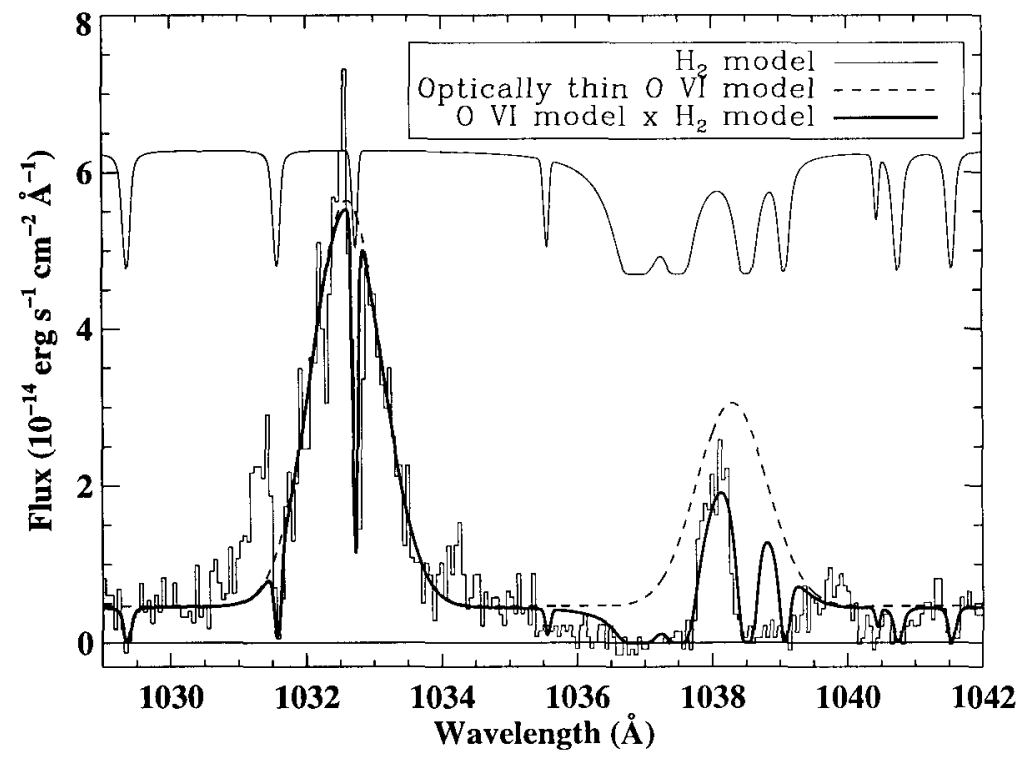

Figure 11. Emission from highly-ionized species in the FUSE spectrum of AB Aur. An $\mathrm{H}_{2}$ absorption model is shown at the top. The optically thin O VI model is shown with a dashed line. The normalized $\mathrm{H}_{2}$ model was multiplied by the $\mathrm{O}$ VI model and overplotted with a thick line. This demonstrates how the strong $\mathrm{H}_{2}$ absorption suppresses the flux from the O vi $\lambda 1038$ line. (From Roberge et al. 2000.) 
FUSE spectra studied by Roberge et al. (2000) reveal many features, ranging from the molecular absorption of $\mathrm{H}_{2}$ and $\mathrm{CO}$ to highly ionized $\mathrm{O}$ VI. In Figure 11 the observed spectrum demonstrates the severe effects of $\mathrm{H}_{2}$ on the emission profile of O VI $\lambda 1038$. The other member of the O VI multiplet has a profile suggestive of complex dynamics. The spectral range of FUSE enables simultaneous study of hot and cold material allowing a probe of stellar magnetic activity, winds, circumstellar material and their interactions.

\subsection{Beta Draconis: a cool supergiant}

Beta Dra (HD 159181) is a luminous cool supergiant (G2 Ib) that can help address the evolution of magnetic activity and the dynamics of stellar winds and mass loss. This star is believed to still possess some large scale magnetic dynamo activity, but is on the brink of decay as its evolution continues to cooler temperatures, and strong winds and mass loss develop. FUSE is important here because the line profiles can give the first indications of mass flow and the onset of winds and mass loss.

The spectrum of $\beta$ Dra (Dupree et al. 2000) shows emission lines from the chromosphere and transition region including: C II, C III, O VI, S III, S IV, S VI, Si III, Si IV, and Fe III. The profile of the $\mathrm{C}$ III $\lambda 977$ line is asymmetric indicative of outwardly moving material. Absorption is present in the profile that may result in part from interstellar material and a natural central reversal of an optically thick line.

\section{The FUSE Hot Star Program}

\section{Presented by A. J. Willis, University College London. ajw@star.ucl.ac.uk}

As part of the FUSE PI Team activity, a major program is being conducted to obtain FUV spectra of a large sample of $O B$ stars (covering all luminosity classes) and WR stars (both WN and WC) in the Galaxy, LMC and SMC - environments which exhibit a range of metallicities. Approximately 120 OB stars and about $30 \mathrm{WR}$ stars will be observed. The FUSE spectral range provides access to P-Cygni profiles (and for WR stars emission lines) of resonance transitions covering a very wide range of ionization potential (e.g. C III, N III, S IV, $\mathrm{S}$ VI, PIV, Pv, and O VI) which are much more amenable to sensitive analysis than the restricted coverage of C IV, Si IV, and NV available to IUE and HST. The FUSE diagnostic transitions, on detailed modelling, will provide more accurate measurements of mass loss rates, wind ionization conditions, and basic stellar parameters (like $T_{\text {eff }}$ and $L_{*}$ ), as well as improved measurements of the nature and character of wind variability phenomena, known to be ubiquitous for massive stars. By observing targets in the three galaxies, the effects of differing galaxy metallicities will be studied, and stellar evolution and mass loss models tested. This paper outlines initial results that have been obtained from the Early Release Observation data.

\subsection{Studies of O-stars in the LMC and SMC}

Fullerton et al. (2000) have reported results from a detailed analysis of two O7 supergiants in the LMC (Sk $-67^{\circ} 111, \mathrm{O} 7 \mathrm{Ib}(\mathrm{f})$ ) and in the SMC (Sk 80, 


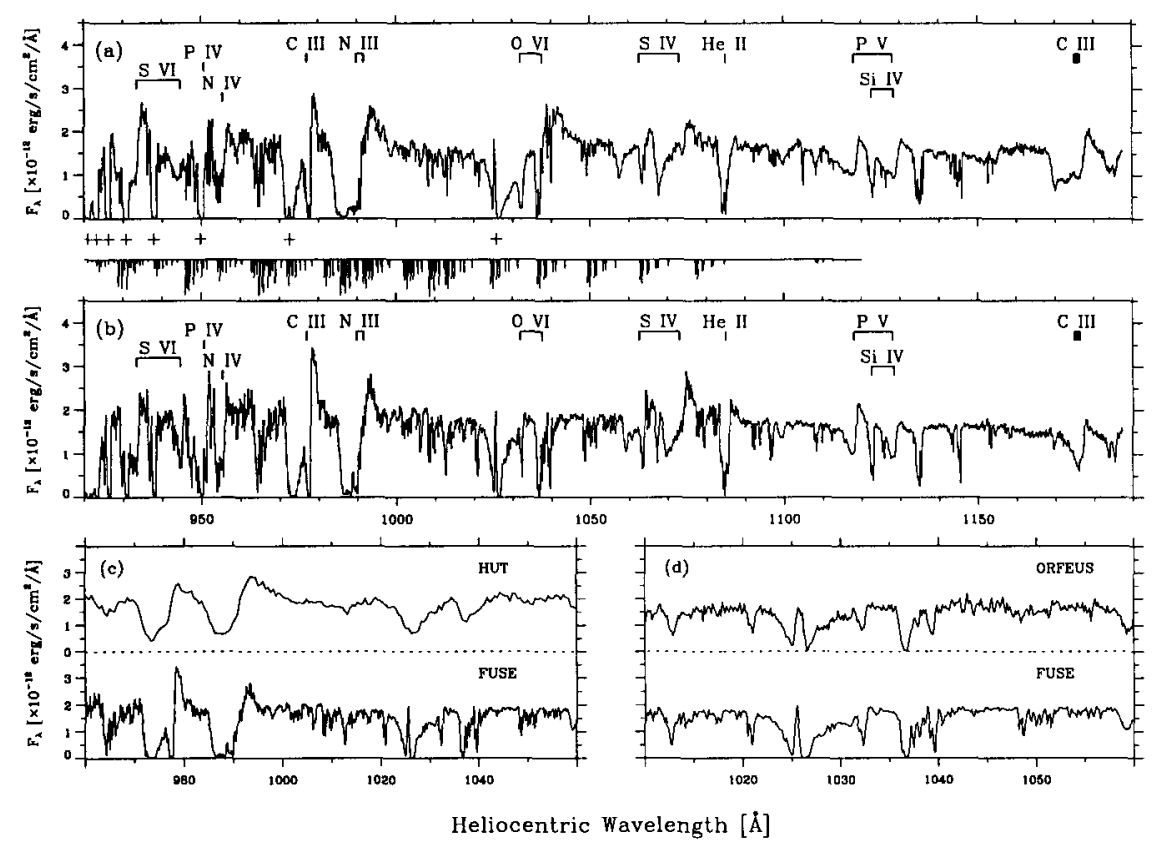

Figure 12. FUSE spectra of two O7 supergiants in the LMC and SMC.

O7 Iaf + ). A comparison of the spectral morphology seen in the plethora of observed P-Cygni profiles, confirms the much lower terminal velocity of the SMC star $\left(v_{\infty}=1400 \mathrm{~km} \mathrm{~s}^{-1}\right)$ compared to the LMC star $\left(v_{\infty}=1800 \mathrm{~km}\right.$ $\mathrm{s}^{-1}$ ), in line with expectations. The SMC star has much weaker O vi P-Cygni absorption (and a much stronger S IV emission - see Fig. 12). Fullerton et al. (2000) have used line-blanketed, hydrodynamic, non-LTE model atmospheres to explore the origin of these differences, and provide improved constraints of the two stellar properties. In the case of Sk $-67^{\circ} 111$ the O VI feature requires a production in shock-heated material in the ambient stellar wind. For both stars, the most striking result is that to fit the observed, strong P-Cygni profiles in CIII, N III, and SIV, a substantially lower value of $T_{\text {eff }}$ may be required than expected from analysis of $I U E / H S T$ and optical spectroscopy. The improved values, with the 'old' values in "()" are: $\mathrm{Sk}-67^{\circ} 111: T_{\text {eff }}=33000 \mathrm{~K}(38000 \mathrm{~K})$ and Sk 80: $T_{\text {eff }}=32000 \mathrm{~K}(37500 \mathrm{~K})$. The resulting stellar luminosities would also be substantially reduced to values of $\log L / L_{\odot}=5.7$ and 5.9 , respectively, roughly a factor of two lower than previously assigned. Subsequent analysis of a larger sample of O9 supergiants in both the LMC and SMC may also indicate lower values of $T_{\text {eff }}$ and luminosity. It is thus becoming apparent that the O star temperature scale may need revision. This may help reconcile the 'mass discrepancy' that has been indicated from analyses of optical spectra and results from binary orbital analyses (see Herreo et al. 2000).

In a complementary study, Bianchi et al. (2000) have used the same spectra, modelled using the simple $S E I$ method, to investigate the ionization balance 
of the stellar winds. They find that the ratios of $\mathrm{SIV} / \mathrm{SVI}$ and $\mathrm{PIV} / \mathrm{PV}$ are consistently lower in the LMC star than in the SMC target. This could be due to the different luminosity and/or different wind densities (resulting from a smaller SMC star mass loss rate). However, the optical depth distribution of ions through the two winds appears to be similar, on taking into account the difference in $v_{\infty}$.

\subsection{The Wolf-Rayet star Sand-2 (WO)}

Crowther et al. (2000) have presented a detailed, quantitative analysis of the FUSE spectra (coupled with HST and ground-based spectra) of the WO star Sand 2 in the SMC. At V = 15.1, this represents one of the faintest stars yet observed with the satellite. The FUSE spectrum is shown in Figure 13, and shows pronounced $\mathrm{P}$-Cygni profiles on $\mathrm{O}$ VI and in numerous $\mathrm{C} I \mathrm{II}$ and $\mathrm{C}$ IV transitions. The measured $v_{\infty}=4100 \mathrm{~km} \mathrm{~s}^{-1}$ is amongst the largest known for a WR star. Detailed non-LTE, line-blanketed modelling of the observed spectra (with results highly constrained by the OVI profile) yield the following stellar parameters: $T_{*}=150,000 \mathrm{~K}, \log L / L_{\odot}=5.3, \dot{M}=1 \times 10^{-5} M_{\odot} \mathrm{yr}^{-1}$, and elemental abundances of $\mathrm{C} / \mathrm{He} \sim 0.7$, and $\mathrm{O} / \mathrm{He} \sim 0.15$. The analysis confirms that Sand 2 is more chemically enriched in carbon than LMC WC4 stars, and is close (i.e. in the next $0.1-5 \times 10^{4} \mathrm{yr}$ ) to undergoing a supernova explosion, as the last phase in the advanced evolution of massive stars, with a likely progenitor initial mass of about $60 M_{\odot}$. As in the case of the $\mathrm{O}$ star analysis, the FUV diagnostic lines (in this case $\mathrm{O}$ VI) provided absolutely crucial constraints on the stellar parameters.

The FUSE hot star program, includes observations of a large sample of WR stars (both of WN and WC types) in the Galaxy and Magellanic Clouds, and from similar modelling techniques can be expected to yield much improved knowledge of the wind parameters, composition and evolutionary status of these objects.

\subsection{Wind variability studies}

Massa et al. (2000) have reported the first results of time-series observations of three Magellanic Cloud O stars, the data being secured during the in-orbit checkout phase, covering time streams of several hours, and intervals of 1-4 months. The targets were: Sk 80 in the SMC, and Sk $-67^{\circ} 05$ and $S k-67^{\circ} 111$ in the LMC. Significantly P-Cygni profile variability is observed in all the stars, with differences apparent in the ionization structure and time scales of the changes from star to star. Wind variability is seen in $\mathrm{Sk}-67^{\circ} 111$ on time scales of less than 10 hours, whilst the coolest star in the sample, Sk $-67^{\circ} 05$, shows the largest variations in O VI. These time-series data highlight the utility of FUSE for this type of investigation, especially spotlighting the importance of unsaturated $\mathrm{P}$-Cygni absorption components in S VI and P V, allowing 'discrete absorption component' structures to be more easily seen. In a Cycle 1 GI program, one LMC star (Sk $-67^{\circ} 166$, O4If) is being studied in great depth through a time-series program spanning observations every day for about 40 days, and a subsequent intensely-sampled series with observations every hour or so. At the time of writing these data have just been secured and are revealing truly remarkable variability patterns in all ions. The detailed analysis of these data 

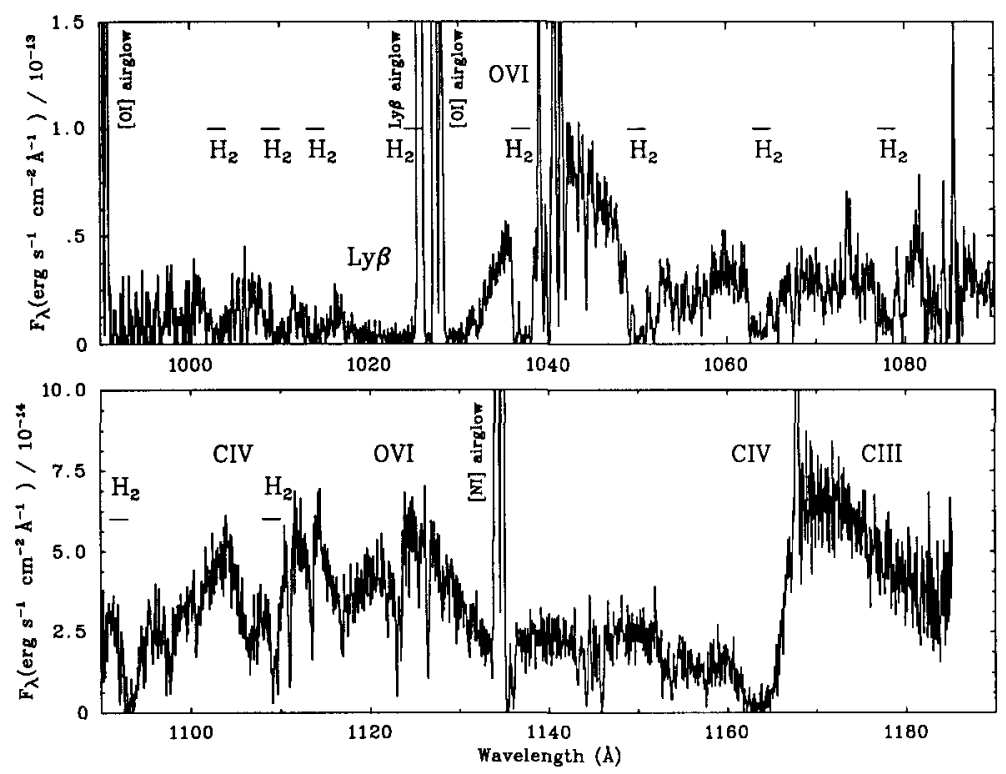

Figure 13. FUSE spectra of the WO star Sand 2.

should provide tight constraints on how the state of the wind structures change as they traverse the wind, their ionization structure, and ultimately on the true physical origin for this phenomena. Progress will be reported by Fullerton et al. (2001).

\section{FUSE Observations of Cataclysmic Variables}

\subsection{The March 2000 Outburst of U Geminorum}

Presented by K. Long, Space Telescope Science Institute. long@stsci.edu

Dwarf novae are mass-exchanging binary systems consisting of a non-magnetic white dwarf (WD) and a relatively normal, lower-mass companion star. The exchange of mass from the secondary star to the WD is mediated by an accretion disk surrounding the white dwarf. In quiescence, the bulk temperature of the disk is low $(<5000 \mathrm{~K})$, mass accumulates in the disk and UV emission in the system arises from the WD and/or a corona on the surface of the disk. However, dwarf novae undergo outbursts, a phase transition of the disk to a hot, optically thick state, during which more mass is lost to the WD and the wind from the disk than is (instantaneously) being accreted from the secondary star. During this period, UV emission is dominated by photons from the disk, some of which are resonantly scattered by a wind driven from the disk. 


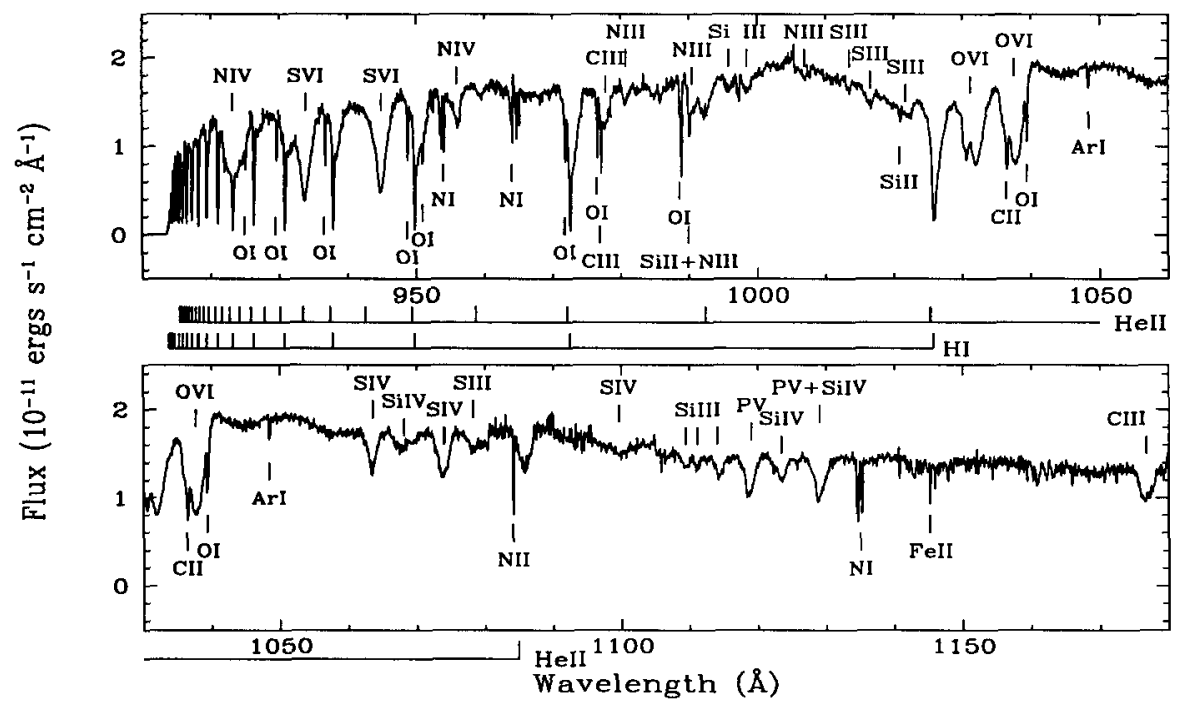

Figure 14. The FUSE FUV spectrum of the dwarf nova U Gem, obtained when the system was at the peak of outburst. Prominent absorption features in the spectrum are labeled.

The goals of our program to observe two dwarf novae - U Geminorum and SS Cygni - with FUSE are to enable us to isolate disk and wind features in the FUV spectrum through an outburst. We plan to use the observations to probe the ionization structure of the disk and the wind as a function of system brightness and to examine the abundances of material in the inner disk and the WD. These two systems were selected because they are extremely well-studied at other wavelengths and because they have quite different wind characteristics. In particular, the optical and ultraviolet wind-features are fairly weak in U Gem compared to SS Cyg.

Here we describe in a preliminary manner the observations of $U$ Gem. We expect to observe SS Cyg in a similar way in the fall of 2000. U Gem underwent a standard "wide" outburst during March 2000. As a result of notification by the American Association of Variable Star Observers and heroic efforts by the FUSE planning team, U Gem was observed four times through the outburst. The first observation took place about 3 days after $\mathrm{U}$ Gem had reached maximum optical brightness. Two additional observations were made during the so-called plateau phase of the outburst. The final observation was made about ten days after the first, at which time the system was in the decline phase $\left(\mathrm{m}_{v} \sim 12.5\right)$ of the outburst. U Gem reached quiescence at $\mathrm{m}_{v}=14.5$, about three days after the last observation.

The time-averaged spectrum from the first observation is shown in Figure 14. The continuum spectrum is characteristic of that expected from a steady-state accretion disk. Comparison of the observed spectrum to synthetic disk spectra constructed from appropriately weighted and doppler-shifted stellar 


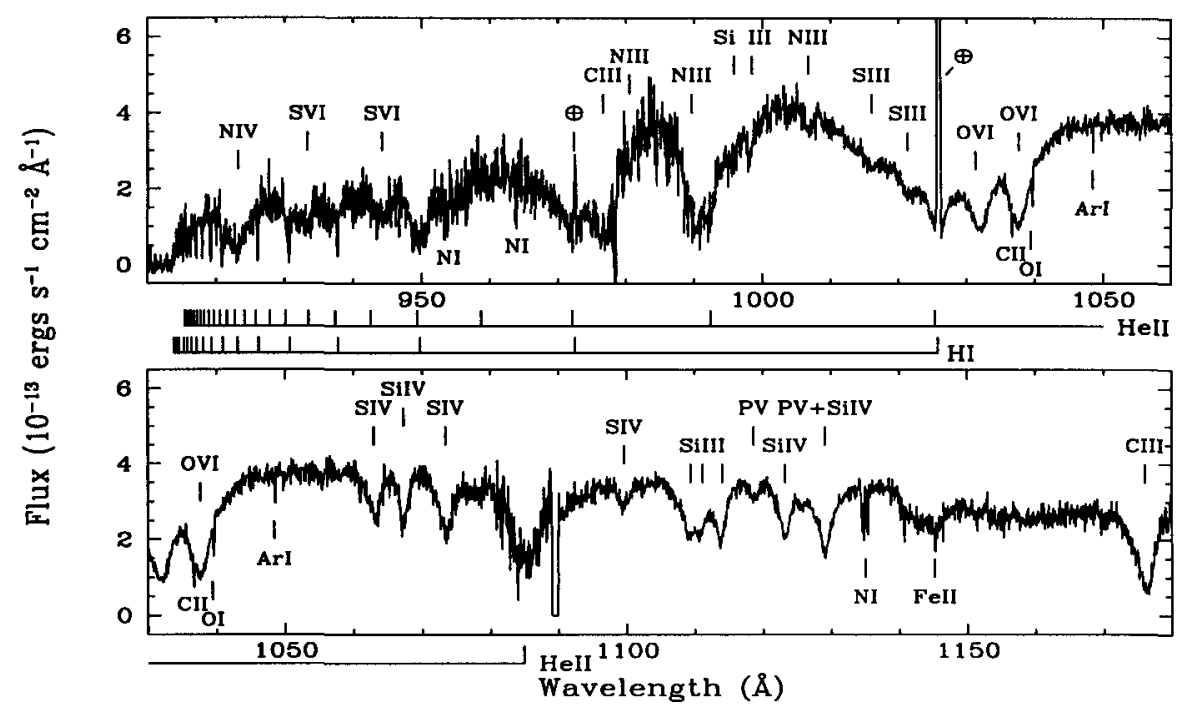

Figure 15. The FUSE FUV spectrum of the dwarf nova U Gem at the end of outburst. This spectrum was obtained 10 days after the spectrum shown in Fig. 14 and three days before the return to quiescence. Prominent absorption lines in the spectrum are labeled.

spectra indicate that the accretion rate was $\sim 10^{18} \mathrm{~g} \mathrm{~s}^{-1}$ and that the flux is consistent with the known distance to U Gem of 95 pc (Harrison et al. 1999). The continuum spectra of this and the other two observations during the plateau phase are punctuated by narrow lines due to interstellar absorption as well as broader absorption features from within U Gem. Prominent features can be identified with lines from CIII, N III, N IV, O VI, Si III, Si IV, S III, S IV, and S VI. The lines have typical velocity widths of $400-600 \mathrm{~km} \mathrm{~s}^{-1}$. Emission wings are generally absent from the lines implying that U Gem does not have the features normally associated with a strong wind. This is consistent with results obtained with HST and IUE (see, e.g., Sion et al. 1997). Line ratios of O vi $\lambda \lambda 1032,1038$ and S VI $\lambda \lambda 933,945$ are 1:1, consistent with optically thick emission partially covering the UV-emitting region of the disk.

Detailed analysis shows that the line centroids track the WD in the system, suggesting that the absorption arises somewhere in the region of outer accretion disk. At extreme ultraviolet (Long et al. 1996) and X-ray (Szkody et al. 1996) wavelengths, the continuum spectra of $U$ Gem is often partially absorbed at near orbital phase 0.7 , an effect that is generally associated with resonances from material in the accretion stream interacting with the disk (see, e.g., Armitage \& Livio 1998). In our plateau-phase observations with FUSE, line strengths vary and are generally stronger near phase 0.6 . The main variation is in the lower ionization state lines, and it is the depth rather than the width of the lines that varies. This is more consistent with an increase in the absorption column or covering fraction than in the velocity widths of the absorbing material. 
As shown in Figure 15, the character of spectrum had changed considerably by the time of our fourth observation. Specifically, the flux has declined to a level very close to that observed in quiescence with HUT in the same wavelength range (Long et al. 1993, 1995) and the shape of the spectrum no longer resembles that of an accretion disk but, instead, is that of a WD. The majority of the lines in the spectrum are those expected in a metal-enriched atmosphere. Evidently, despite the fact that the optical magnitude of the system is still about 2 magnitudes above quiescence, FUV emission from the disk is nearly absent. Preliminary attempts to model the spectrum indicate an average WD surface temperature of $\sim 43,000 \mathrm{~K}$ and a WD radius at $95 \mathrm{pc}$ of $\sim 3.8 \times 10^{8} \mathrm{~cm}$, similar to, but slightly smaller than expected for a $1.2 M_{\odot}$ WD. The temperature is near to that observed with HUT 11 days after a wide outburst in 1990.

Our preliminary analyses of the U Gem spectrum already show the richness of the information we expect to derive. Attempts to locate the position of the absorption lines using a Monte Carlo radiative transfer code (Long \& Knigge 1998) are underway; preliminary results are encouraging but require material quite far from the disk plane (given that the inclination of the system is near $67^{\circ}$; Long \& Gilliland 1999). We are also using this code to explore the possibility that the absence of emission wings on strong resonance lines in the outburst is due not to the absence of a wind but to ionization of the wind by the boundary layer emission seen with EUVE (Long et al. 1996). We also look forward to the completion of our Cycle 1 GI program, which includes a similar exciting observing sequence for SS Cyg.

\subsection{The Magnetic Polar BY Cam}

Presented by M.Mouchet, Observatoire de Paris-Meudon, martine.mouchet@obspm.fr

The cataclysmic binary BY Cam belongs to the class of polars that are semidetached systems composed of a strongly magnetized white dwarf $\left(B \sim 10^{7}-10^{8}\right.$ G) accreting matter from a red dwarf filling its Roche lobe and whose rotation is synchronized with the orbital motion. Close to the white dwarf, accretion occurs along the magnetic field lines and results in strong X-ray emission. The energy budget is dominated by the release of the accretion energy. Strong emission lines superimposed on a blue continuum are present in the UV and optical spectra. BY Cam is peculiar in several respects, with a high hard-to-soft X-ray ratio (Kallman et al. 1996) and being slightly desynchronized (Silber et al. 1992). In addition IUE observations of BY Cam revealed that the emission resonance line ratio $\mathrm{N} \mathrm{V} / \mathrm{C} \mathrm{IV}$ is more than ten times larger than what is normally observed in polars (Bonnet-Bidaud \& Mouchet 1987, or BM87). The FUSE observations have given access for the fist time to the resonance O VI line for evaluating CNO abundances.

BY Cam was observed on 10 and 16 January 2000, but since the first observation was affected by bad centering in the aperture, only the 16 Jan. data are presented below. The dataset consists of five exposures in the large aperture, resulting in a total $22 \mathrm{ksec}$ on-source exposure time, nearly covering all target orbital phases. These observations were simultaneous with RXTE and EUVE 


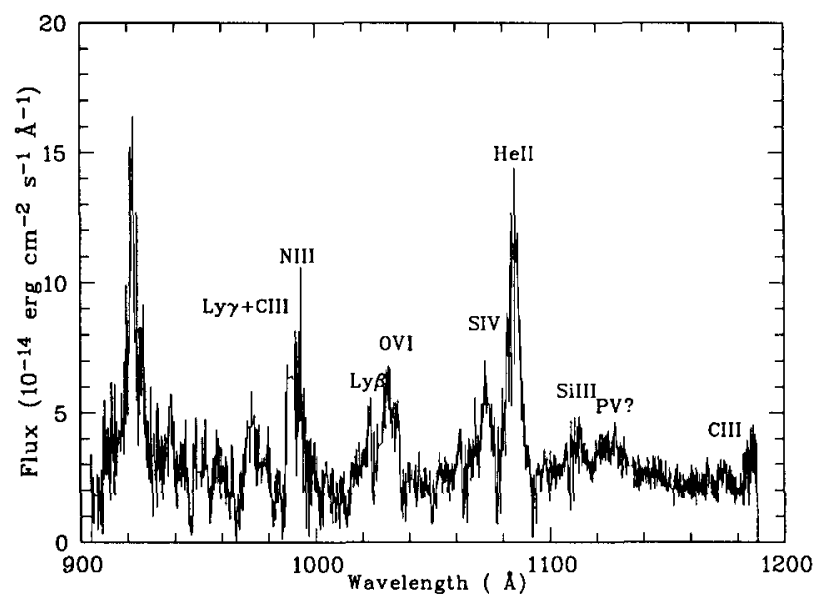

Figure 16. Average spectrum of BY Cam after removing the geocoronal lines. Data have been binned over $0.1 \AA$. The feature at $920 \AA$ is most probably a residual of geocoronal lines.

observations as well as spectroscopy and photometry from several ground-based telescopes.

The average spectrum obtained by combining all detectors and exposures is shown in Figure 16. The strongest geocoronal lines have been removed. The continuum flux is in agreement with the high state IUE spectrum and can be described with a power law $F_{\lambda} \propto \lambda^{-1.43}$ if no reddening is assumed $(-2.44$ for $E_{\mathrm{B}-\mathrm{V}}=0.05$ ). Broad emission lines are detected including the hydrogen Lyman series, the resonance doublet $\mathrm{O}$ VI and strong lines of $\mathrm{He}$ II and $\mathrm{N}$ III. The equivalent widths of the strongest $\mathrm{H}_{2}$ lines indicate a column density larger than $10^{19} \mathrm{~cm}^{-2}$.

Contrary to AM Her, no narrow O VI component is detected in BY Cam. The intensity has been derived by fitting two gaussians of same widths, at the doublet separation, assuming $N\left(\mathrm{H}_{2}\right)=10^{19} \mathrm{~cm}^{-2}$. The resulting $\mathrm{O}$ VI equivalent width is about half that of the broad component of AM Her (Mauche \& Raymond 1998), with a doublet intensity ratio of 1.2 . The ratios $\mathrm{N} \mathrm{V} / \mathrm{C} I V$ and $\mathrm{O}$ VI/C IV are 5.1 and 0.70 for BY Cam (0.12 and 0.34 for AM Her). For AM Her, values are from ORFEUS (Mauche \& Raymond 1998) and HUT spectra (Greeley et al. 1999) and refer to the broad component only.

The broad resonance lines of the highly ionized species of $\mathrm{C} \mathrm{IV}, \mathrm{N} V$ and $\mathrm{O}$ VI are expected to be formed mainly in the accreting column, photoionized by the $\mathrm{X}$-ray flux. The resonance line ratios have been compared to values obtained from the CLOUDY plasma code (Ferland et al. 1998) for different values of the ionisation parameter $U=\frac{Q(1 R y d-100 \mathrm{MeV})}{4 \pi R^{2}} \cdot \frac{1}{n_{H} c}$ where $Q$ is the number of hydrogen-ionizing photons emitted by the central source at a distance $R$ from the illuminated medium. Two ionizing spectral shapes have been considered, a $30 \mathrm{eV}$ blackbody and a $10 \mathrm{keV}$ bremsstrahlung, which are typical of what is observed in polars. A density of $n_{H}=10^{12} \mathrm{~cm}^{-3}$ and a column density of $N(\mathrm{H} \mathrm{I})=10^{21} \mathrm{~cm}^{-2}$ are assumed. Ionic fractions of $\mathrm{O} \mathrm{VI}, \mathrm{NV}$ and $\mathrm{C}$ IV greater 
than 0.1 are obtained for $U$ values greater than $5 \times 10^{-3}$ for both cases of illuminating spectra. For AM Her typical line ratios are roughly reproduced for $U \sim 0.01$ while values for BY Cam cannot be accounted for.

More adequate computations have been performed by adopting parameter values determined for BY Cam. The ionizing spectrum was defined as the sum of a $20 \mathrm{keV}$ bremsstrahlung, as observed in the RXTE observations, and of a $50 \mathrm{eV}$ blackbody with a bolometric luminosity of 0.1 times the hard X-ray luminosity (Ramsay et al. 1994). Assuming a dipole geometry and free-fall velocities along the accretion column (cf. Stockman \& Schmidt 1996), the density varies with the distance to the white dwarf as $R^{-2.5}$ and the parameter $U$ increases as $R^{+0.5}$, resulting in values of $U$ in the range $(0.6-4) \times 10^{-3}$ for distances from $5 \times 10^{9}$ to $5 \times 10^{10} \mathrm{~cm}$. These values are too low to produce sufficient fractions of CIV, $\mathrm{NV}$ and $\mathrm{OVI}$. The presence of a non-detected additional contribution to the ionizing flux, such as a very soft $\mathrm{X}$-ray component, would produce higher values of $U$. This hidden component should be compatible with the flux upper limit at $100 \mathrm{eV}$ of $6 \times 10^{-15} \mathrm{erg} / \mathrm{s} / \mathrm{cm}^{2} / \AA$ derived from the EUVE observations. For a column density of $2 \times 10^{20} \mathrm{~cm}^{-2}$, in agreement with the color excess derived from the IUE spectra, a $10 \mathrm{eV}$ blackbody component with a luminosity similar to that of the bremsstrahlung, would not be detected and would give $U$ values as large as 0.1 . However the observed resonance line ratios still cannot be reproduced.

Previous computations have been done for solar abundances. However, the strong lines of N III $990 \AA$ and N IV $1718 \AA$ indicate an overabundance of nitrogen, while an underabundance of carbon is suggested by the weaknesses of $\mathrm{C}$ III $977 \AA$ and $1175 \AA$. Such non-solar values might be the consequence of a nova-type explosion though the recent nova V1500 Cyg does not show abnormal UV line intensities and the ejecta observed in a large sample of novae show enrichment for all three elements C, N, and O (Livio \& Truran 1994). Enhancement of N and depletion of $\mathrm{C}$ can be obtained in the context of evolution models of semidetached systems in which the secondary surface is polluted by re-accretion of material ejected during nova outburts (Marks \& Sarna 1998).

Stronger constraints on the abundance values should be obtained from the $\mathrm{H}$-like and He-like lines of $\mathrm{C}, \mathrm{N}$, and $\mathrm{O}$ which are accessible with XMM-Newton and Chandra. High spectral resolution N V and C IV line profiles could provide more insight on the components originating from the accretion column.

Acknowledgments. The work presented in this paper is based on observations made with the NASA-CNES-CSA Far Ultraviolet Spectroscopic Explorer. FUSE is operated for NASA by the Johns Hopkins University under NASA contract NAS5-32985.

\section{References}

Ake, T. B., et al. 2000, ApJ, 538, L87

Akeson, R. L. \& Blitz, L. 1999, ApJ, 523, 163

Allen, M. M. 1994, ApJ, 424, 754

Armitage, P. J. \& Livio, M. 1998, ApJ, 493, 898

Audouze, J., Tinsley, B. M., 1976, ARA\&A, 14, 43

Bechtold, J., Weyman, R. J., Lin, Z., \& Malkan, M. A. 1987, ApJ, 315, 180 
Bechtold, J. 1993, in The Environment and Evolution of Galaxies, ed. J. M. Shull \& H. A. Thronson, Kluwer, p 559

Berghöfer, T. W., Bowyer, S., Lieu, R., \& Knude, J. 1998, in The Local Bubble and Beyond, ed. D. Breitschwerdt, M. J. Freyberg, \&J. Trümper (Berlin: Springer), p. 61

Bianchi, L., et al. 2000, ApJ, 538, L57

Bland-Hawthorn, J. et al. 1998, MNRAS, 299, 611

Blitz, L., Spergel, D., Teuben, P., Hartmann, D., \& Burton, W. B. 1999, ApJ, 514,818

Boesgaard, A.M., Steigman, G., 1985, ARA\&A, 23, 319

Bonnet-Bidaud, J.M., Mouchet M., 1987, A\&A, 188, 89

Bookbinder, J., Walter, F., \& Brown, A. 1992, in ASP Conf. Ser. 26, Cambridge Workshop on Cool Stars, Stellar Systems, and the Sun, ed. M. S. Giampapa \& J. A. Bookbinder (San Francisco: ASP), 27

Bregman, J. 1980, ApJ, 236, 577

Breitschwerdt, D., \& Schmutzler, T. 1994, Nat., 371, 774

Burles, S., Tytler, D. 1998a, ApJ, 499, 699

Burles, S., Tytler, D. 1998b, ApJ, 507, 732

Burles, S., et al. 1999, Phys.Rev.Lett, 82, 4176

Butler, S. E., \& Dalgarno, A. 1979, ApJ, 234, 765

Carruthers, G. 1970, ApJ, 161, L81

Cassé, M., \& Vangioni-Flam, E. 1998, in Structure and Evolution of the Intergalactic Medium from QSO Absorption Line Systems, ed. Petitjean, P., \& Charlot, S., 331

Cassé, M., Vangioni-Flam, E. 2000, in Third Integral Workshop (ESA), Taormina, Sept. 1998, in press

Collier Cameron, A. et al. 1999, MNRAS, 308, 493

Cox, D. P., \& Reynolds, R. J. 1987, ARA\&A, 25, 303

Crenshaw. D. M., Kraemer, S. B., Boggess, A., Maran, S. P., Mushotzky, R. F., \& Wu, C. C. 1999, ApJ, 516, 750

Crowther, P.A., et al. 2000, ApJ, 538, L51

de Boer, K. S., Richter, P., Bomans, D. J., Heithausen, A., \& Koornneef, J. 1998, A\&A, 338, L5

Dove, J. B., Shull, J. M. \& Ferrara, A. 2000, ApJ, 531, 846

Dupuis, J., Vennes, S., Bowyer, S., Pradhan, A. K., \& Thejll, P. 1995, ApJ, 455, 574

Dupree, A. K., \& Raymond, J. C. 1983, ApJ, 275, L71

Dupree, A. K., Young, P. R., \& Ake, T. B. 2000, BAAS, in press.

Edgar, R. J., \& Savage, B. D. 1989, ApJ, 340, 762

Fardal, M. A. Giroux, M. L., \& Shull, J. M. 1998, AJ, 115, 2206

Federman, S. R., Cardelli, J. A., van Dishoeck, E. F., Lambert D. L. \& Black, J. H. 1995, ApJ, 445, 325 
Federman, S. R., Strom, C. J., Lambert, D. L., Cardelli, J. A., Smith, V. V. \& Joseph, C. L. 1994, ApJ, 424, 772

Ferland, G.J., Korista, K.T., Verner, D.A., et al. 1998, PASP, 110, 761

Ferlet, R. 1981, A\&A, 98, L1

Ferlet, R. 1999, A\&A Rev., 9, 153

Field, G. B., \& Steigman, G. 1971, ApJ, 166, 59

Fransson, C., \& Chevalier, R. A. 1985, ApJ, 296, 35

Frisch, P. C. 1995, Space Sci. Rev., 72, 499

Fullerton, A.W., et al. 2000, ApJ, 538, L43

Fullerton, A.W., Massa, D.., Willis, A.J., Howarth, I.D., Prinja, R.K., 2001, in preparation

Gardiner, L. T. 1999, in ASP Conf. Series 166, The Stromlo Workshop on High Velocity Clouds, ed. B.K. Gibson \& M.E. Putman, (San Francisco: ASP), 292

George, I. M., Turner, T. J., Netzer, H., Nandra, K., Mushotzky, R. F., \& Yaqoob, T. 1998, ApJS, 114, 73

Giallongo, E., Fontana, A. \& Madau, P. 1997, MNRAS, 289, 629

Gibson, B. K., Giroux, M. L., Penton, S. L., Stocke, J. T., \& Shull, J. M. 2000, AJ, 120,1830

Grady, C. A., Woodgate, B., Bruhweiler, F. C., Boggess, A., Plait, P., Lindler, D. J. Clampin, M., \& Kalas, P. 1999, ApJ, 523, L151

Greeley B., Blair, W., Long, K., Raymond J., 1999, ApJ, 513, 491

Gry, C. 1996, Space Sci. Rev., 78, 239

Haardt, F. \& Madau, P. 1996, ApJ, 461, 20

Harrison, T. E., McNamara, B. J., Szkody, P., McArthur, B. E., Benedict, G. F., Klemola, A. R. \& Gilliland, R. L. 1999, ApJ, 515, L93

Hartquist, T. W., Pettini, M., \& Tallant, A. 1984, ApJ, 276, 519

Heiles, C. 1984, ApJS, 55, 585

Herreo, A., Puls, J., \& Villamariz, M.R., 2000, A\&A, 354, 193

Hester, J. J., et al. 1996, AJ, 111, 2349

Hurwitz, M., Jelinsky, P. \& Dixon, W. V. D. 1997, ApJ, 481, L31

Hurwitz, M., \& Bowyer, S. 1996, ApJ, 446, 812

Hurwitz, M., et al. 1998a, ApJ, 500, L1

Hurwitz, M., et al. 1998b, ApJ, 500, L61

Ito, M., \& Ikeuchi, S. 1988, PASJ, 40, 403

Jaffe, A. II., et al. 2000, astro-ph/0007333

Jenkins, E. B. 1978a, ApJ, 219, 845

Jenkins, E. B. 1978b, ApJ, 220, 107

Jenkins, E. B., Gry, C., \& Dupin, O. 2000, A\&A, 354, 253

Jenkins, E. B., Lees, J. F., van Dishoeck, E. F., \& Wilcots, E. M. 1989, ApJ, 343,785 
Jenkins, E. B., Tripp, T. M., Woźniak, P. R., Sofia, U. J., Sonneborn, G. 1999, ApJ, 520, 182

Jenkins, E. B., et al. 2000, ApJ, 538, L81

Kaastra, J. S., Mewe, R., Liedahl, D. A., Komossa, S., \& Brinkman, A. C. 2000, A\&A, 354, L931

Kallman T.R., Mukai, K., Schlegel, E.M., et al. 1996, ApJ, 466, 973

Kaplinghat, M., Turner, M.S. 2000, astro-ph/0007454

Kaspi, S., Brandt, W. N., Netzer, H., Sambruna, R., Chartas, G., Garmire, G. P., \& Nousek, J. A. 2000, ApJ, 535, L17

Kriss, G. A., Davidsen, A. F., Zheng, W., Kruk, J. W., \& Espey, B. R. 1995, ApJ, 454, L7

Kriss, G. A., et al. 1992, ApJ, 392, 485

Kriss G. A., et al. 1996a, ApJ, 467, 622

Kriss G. A., et al. 1996b, ApJ, 467, 629

Kriss, G. A., Peterson, B. M., Crenshaw, D. M., \& Zheng, W. 2000a, ApJ, 535, 58

Kriss, G. A., et al. 2000b, ApJ, 538, L17

Kruk, J. W., et al. 1995, ApJ, 454, L1

Lallement, R. 1996, Space Sci. Rev., 78, 361

Lallement, R. 1998, in Proc. IAU Colloq. 166: The Local Bubble and Beyond, ed. D. Breitschwerdt, M. J. Freyberg, \& J. Trümper (Berlin: Springer), p. 19

Lallement, R., \& Bertin, P. 1992, A\&A, 266, 479

Lallement, R., \& Ferlet, R. 1997, A\&A, 324, 1105

Lemoine, M., et al. 1996, A\&A, 308, 601

Lemoine, M., et al. 1999, New. Astron., 4, 231

Leitherer, C., Ferguson, H. C., Heckman, T. M. \& Lowenthal, J. D. 1995, ApJ, 454, L19

Lin, D. N. C., Jones, B. F., \& Klemola, A. R. 1995, ApJ, 439, 652

Linsky, J. L. 1996, Space Sci. Rev., 78, 157

Linsky, J., et al. 1995, ApJ, 451, 335

Livio, M., Truran, J.W., 1994, ApJ, 425, 797

Long, K. S., Blair, W. P. \& Raymond, J. C. 1995, ApJ, 454, L39

Long, K. S., et al. 1993, ApJ, 405, 327

Long, K., et al. 1996, ApJ, 469, 841

Long, K. S. \& Gilliland, R. L. 1999, ApJ, 511, 916

Long, K. S. \& Knigge, C. 1998, AAS Meeting, 193, 110305

Lu, L., et al. 1998, AJ, 115, 162

Lyu, C. H., \& Bruhweiler, F. C. 1996, ApJ, 459, 216

Madau, P. \& Shull, J. M. 1996, ApJ, 457, 551

Marks, P.B., Sarna, M.J., 1998, MNRAS, 301, 699

Marscher, A. P., Moore, E. M. \& Bania, T. M. 1993, ApJ, 419, L101 
Massa, D., et al. 2000, ApJ, 538, L47

Mathur, S., Wilkes, B., \& Elvis, M. 1995, ApJ, 452, 230

Mathur, S., Wilkes, B., Elvis, M., \& Fiore, F. 1994, ApJ, 434, 493

Mauche, C.W., Raymond J.C., 1998, ApJ, 505, 869

McLachlan, A. \& Nandy, K. 1984, MNRAS, 207, 355

Meyer, D. M., Jura, M., \& Cardelli, J. A. 1996, BAAS, 28, 892

Meyer, D. M., Cardelli, J. A., Sofia, U. J. 1997, ApJ, 490, L103

Meyer, D. M., Jura, M., Cardelli, J. A. 1998, ApJ, 493, 222

Mirabel, I. F. \& Morras, R. 1984, ApJ, 279, 86

Moore, B. \& Davis, M. 1994, MNRAS, 270, 209

Moos, H. W., et al. 2000, ApJ, 538, L1

Murphy, E. M., et al. 2000, ApJ, 538, L35

Nollett, K.M., Burles, S. 2000, Phys.Rev.D, in press (astro-ph/0001440)

Olive, K., et al. 1990, Phys.Rev.Lett, B236, 454

Oort, J. H. 1970, A\&A, 7, 381

Pagel, B., et al. 1992, MNRAS, 255, 325

Penton, S. V., Shull, J. M., \& Stocke, J. 2000, ApJ, 544, 150

Prantzos, N. 1996, A\&A, 310, 106

Putman, M. E. \& Gibson, B.K. 1999, PASA, 16, 70

Putman, M. E., et al. 1998, Nat., 394, 752

Quémerais, E., Bertaux, J.-L., Sandel, B. R., \& Lallement, R. 1994, A\&A, 290, 941

Ramsay, G., Mason, K., Cropper, M., et al. 1994, MNRAS, 270, 692

Redfield, S., \& Linsky, J. L. 2000, ApJ, 534, 825

Reynolds, C. S. 1997, MNRAS, 286, 513

Richter, P. de Boer, K. S., Widmann, H., Kappelmann, N., Gringel, W., Grewing, M., \& Barnstedt, J. 1999, Nat., 402, 386

Richter, P., Savage, B. D., Wakker, B. P., Sembach, K. R., \& Kalberla, P. M. W. 2000, ApJ, in press

Roberge, A., et al. 2000, ApJ, 551, L97

Rogerson, J., York, D. 1973, ApJ, 186, L95

Sahnow, D. J., et al. 2000, ApJ, 538, L7

Sahu, M. S., et al. 1999, ApJ, 523, L159

Savage, B. D., Sembach, K.R., \& Lu, L. 1997, AJ, 113, 2158

Savage, B. D., \& Sembach, K.R. 1991, ApJ, 379, 245

Savage, B. D., et al. 2000, ApJ, 538, L27

Scully, S. T., Cassé, M., Olive. K. A., Vangioni-Flam, E. 1997, ApJ, 476, 521

Sembach, K. R., Howk, J. C., Savage, B. D., \& Shull, J. M. 2001, ApJ, 561, 573

Sembach, K. R., Savage, B. D., \& Hurwitz, M. 1999, ApJ, 524, 98

Sembach, K. R., Savage, B. D., \& Tripp, T. M. 1997, ApJ, 480, 216

Sembach, K. R., Savage, B. D., Lu, L., \& Murphy, E. M. 1995, ApJ, 451, 616 
Sembach, K. R., Savage, B. D., Lu, L., \& Murphy, E. M. 1999, ApJ, 515, 108

Sembach, K. R., et al. 2000, ApJ, 538, L31

Sfeir, D. M., Lallement, R., Crifo, F., \& Welsh, B. Y. 1999, A\&A, 346, 785

Shapiro, P.R., \& Field, G. B. 1976, ApJ, 205, 762

Shapiro, P. R., \& Moore, R. T. 1976, ApJ, 207, 460

Shull, J. M., et al. 2000a, ApJ, 538, L13

Shull, J. M., et al. 2000b, ApJ, 538, L73

Shull, J. M., Roberts, D., Giroux, M. L., Penton, S. V. \& Fardal, M. A. 1999, AJ, 118, 1450

Shull, J. M., \& Slavin, J. D. 1994, ApJ, 427, 784

Silber, A., Bradt, H.V., Ishida, M. et al. 1992, ApJ, 389, 704

Sion, E. M. et al. 1997, ApJ, 483, 907

Slavin, J. D. 1989, ApJ, 346, 718

Slavin, J. D., Shull, J. M., \& Begelman, M. C. 1993, ApJ, 407, 83

Snow, T. P., et al. 2000, ApJ, 538, L65

Snowden, S. L. 1998, in The Local Bubble and Beyond, ed. D. Breitschwerdt, M. J. Freyberg, \& J. Trümper (Berlin: Springer), p.103

Snowden, S. L., Cox, D. P., McCammon, D., \& Sanders, W. T. 1990, ApJ, 354, 211

Snowden, S. L., Freyberg, M. J., Kuntz, K. D., \& Sanders, W. T. 2000, ApJS, 128,171

Sofia, U. J., \& Jenkins, E. B. 1998, ApJ, 499, 951

Songaila, A., Cowie, L. L. \& Lilly, S. J. 1990, ApJ, 348, 371

Sonneborn, G., et al. 2000, ApJ, 545, 277

Spitzer, L. 1956, ApJ, 124, 20

Spitzer, L. 1990, ARA\&A, 28, 71

Spitzer L. 1996, ApJ, 458, L29

Spitzer, L., \& Jenkins, E. B., 1975, ARA\&A, 13, 133

Steidel, C. C., Pettini M. \& Adelberger, K. L. 2000, ApJ, 546, 665

Stockman, H.S., Schmidt, G.D. 1996, ApJ, 468, 883

Sutherland, R. S., \& Dopita, M. A. 1993, ApJS, 88, 253

Szkody, P., Long, K. S., Sion, E. M. \& Raymond, J. C. 1996, ApJ, 469, 834

Tripp, T., \& Savage, B. D. 2000, ApJ, 542, 42

Tripp, T., Savage, B. D., \& Jenkins, E. B. 2000, ApJ, 534, L1

Tufte, S. L., Reynolds, R. J., \& Haffner, L. M. 1998, ApJ, 504, 773

Tumlinson, J., Giroux, M. L., Shull, J. M. \& Stocke, J. T. 1999, AJ, 118, 2148

Tumlinson, J., et al. 2001, ApJ, in press

Tytler, D., et al. 1999, AJ117, 63

Vallerga, J. 1998, ApJ, 497, 921

Vangioni-Flam, E., Cassé, M. 1994, ApJ, 427, 618

Vangioni-Flam, E., Cassé, M. 1995, ApJ, 441, 471 
Vidal-Madjar, A., et al. 1998, A\&A, 338, 694

Vidal-Madjar, A. 2000, in "The Light Elements and Their Evolution" , Natal, November 1999, ASP Conference Series, eds. L. da Silva \& J. R. de Medeiros, in press

Vilhu, O., Muhli, P., Huovelin, J., Hakala, P., Rucinski, S. M., \& Collier Cameron, A. 1998, AJ, 115, 1610

Wakker, B. P., Murphy, E. M., van Woerden, H., \& Dame, T.M. 1997, ApJ, 488,216

Wakker, B. P. \& van Woerden, H. 1997, ARA\&A, 35, 217

Wakker, B. P., et al. 1999a, Nat., 402, 388

Wakker, B. P., Savage, B. D., Oosterloo, T. A., \& Putman, M. E. 1999b, in ASP Conf. Series 166, The Stromlo Workshop on High Velocity Clouds, ed. B. K. Gibson \& M. E. Putman, (San Francisco: ASP), 302

Webb, J.K., et al. 1997, Nat. 388, 250

Welsh, B. Y., Sfeir, D. M., Sirk, M. M., \& Lallement, R. 1999, A\&A, 352, 308

Widmann, H., et al. 1999, A\&A, 338, L1

Williamson, F. O., Sanders, W. T., Kraushaar, W. L., McCammon, D., Borken, R., \& Bunner, A. N. 1974, ApJ, 193, L133

Wolff, B., Koester, D., \& Lallement, R. 1999, A\&A, 346, 969

Wood, B. E., \& Linsky, J. L. 1997, ApJ, 474, L39

York, D. G. 1974, ApJ, 193, L127

York, D. G., et al. 1983, ApJ, 266, L55

Young, P. R., Dupree, A. K., \& Kenyon, T. B. 2000, BAAS, in press.

Zheng, W., Kriss, G. A., Telfer, R. C., Grimes, J. P., \& Davidsen, A. F. 1997, ApJ, 475, 469 